\title{
High salt primes a specific activation state of macrophages, $\mathrm{M}(\mathrm{Na})$
}

Wu-Chang Zhang ${ }^{1, *}$, Xiao-Jun Zheng ${ }^{1, *}$, Lin-Juan Du ${ }^{1, *}$, Jian-Yong Sun ${ }^{1}$, Zhu-Xia Shen ${ }^{1}$, Chaoji Shi ${ }^{2}$, Shuyang Sun ${ }^{2}$, Zhiyuan Zhang ${ }^{2}$, Xiao-qing $\mathrm{Chen}^{3}$, Mu Qin ${ }^{3}$, Xu Liu ${ }^{3}$, Jun Tao ${ }^{4}$, Lijun Jia ${ }^{5}$, Heng-yu Fan ${ }^{6}$, Bin Zhou ${ }^{1}$, Ying Yu ${ }^{1}$, Hao Ying ${ }^{1}$, Lijian Hui ${ }^{7}$, Xiaolong Liu ${ }^{7}$, Xianghua Yi ${ }^{8}$, Xiaojing Liu', Lanjing Zhang ${ }^{10,11,12,13}$, Sheng-Zhong Duan ${ }^{1}$

${ }^{1}$ Key Laboratory of Nutrition and Metabolism, Institute for Nutritional Sciences, Shanghai Institutes for Biological Sciences, Chinese Academy of Sciences, University of the Chinese Academy of Sciences, Shanghai 200031, China; ${ }^{2}$ Shanghai Key Laboratory of Stomatology, Department of Oral and Maxillofacial-Head Neck Oncology, Ninth People's Hospital, Shanghai Jiao Tong University School of Medicine, Shanghai 200011, China; ${ }^{3}$ Department of Cardiology, Shanghai Chest Hospital, Shanghai Jiao Tong University, Shanghai 200030, China: ${ }^{4}$ Department of Hypertension and Vascular Disease, The First Affiliated Hospital, Sun YatSen University, Guangzhou, Guangdong 510080, China; ${ }^{5}$ Cancer Institute, Fudan University Shanghai Cancer Center, Fudan University, Shanghai 200032, China; ${ }^{6}$ Life Sciences Institute and Innovation Center for Cell Biology, Zhejiang University, Hangzhou, Zhejiang 310058, China; ' State Key Laboratory of Cell Biology, Institute of Biochemistry and Cell Biology, Shanghai Institutes for Biological Sciences, Chinese Academy of Sciences, Shanghai 200031, China; ${ }^{8}$ Department of Pathology, Tongji Hospital, Tongji University School of Medicine, Shanghai 200065, China; ${ }^{9}$ Laboratory of Cardiovascular Diseases, Regenerative Medicine Research Center, West China Hospital, Sichuan University, Chengdu, Sichuan 610041, China; ${ }^{10}$ Department of Pathology, University

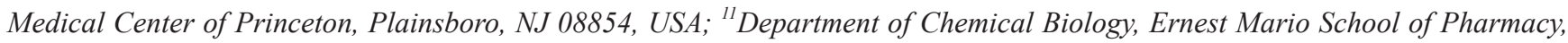
Rutgers University, Piscataway, NJ 08854, USA; ${ }^{12}$ Department of Pathology, Robert Wood Johnson Medical School, Rutgers University, Piscataway, NJ 08854, USA; ${ }^{13}$ Cancer Institute of New Jersey, Rutgers University, Piscataway, NJ 08854, USA

High salt is positively associated with the risk of many diseases. However, little is known about the mechanisms. Here we showed that high salt increased proinflammatory molecules, while decreased anti-inflammatory and proendocytic molecules in both human and mouse macrophages. High salt also potentiated lipopolysaccharide-induced macrophage activation and suppressed interleukin 4-induced macrophage activation. High salt induced the proinflammatory aspects by activating p38/cFos and/or Erk1/2/cFos pathways, while inhibited the anti-inflammatory and proendocytic aspects by Erk1/2/signal transducer and activator of transcription 6 pathway. Consistent with the in vitro results, high-salt diet increased proinflammatory gene expression of mouse alveolar macrophages. In mouse models of acute lung injury, high-salt diet aggravated lipopolysaccharide-induced pulmonary macrophage activation and inflammation in lungs. These results identify a novel macrophage activation state, $\mathrm{M}(\mathrm{Na})$, and high salt as a potential environmental risk factor for lung inflammation through the induction of $\mathrm{M}(\mathrm{Na})$.

Keywords: high salt; macrophage activation; acute lung injury

Cell Research (2015) 25:893-910. doi:10.1038/cr.2015.87; published online 24 July 2015

\section{Introduction}

\footnotetext{
*These three authors contributed equally to this work. Correspondence: Sheng-Zhong Duan

Tel/Fax: +86-21-5492-0981

E-mail: szduan@sibs.ac.cn

Received 11 March 2015; revised 12 May 2015; accepted 30 May 2015; published online 24 July 2015
}

Sodium is an indispensable nutrient and an appropriate amount of it in the body is critical for proper cellular functions of living animals [1]. Sodium is essential for maintenance of osmotic pressure, distribution of body fluids, normal $\mathrm{pH}$ and most metabolic processes. The influx of sodium ions across plasma membrane is required for action potential that is fundamental for nerve impulses and muscle contraction. Too little sodium is 
insufficient to maintain these important functions. On the contrary, sodium is readily available and consumed in large amounts from diets in modern societies [2], posing severe threats to public health [3-5]. However, how excessive amounts of sodium cause health problems remains poorly understood.

Immune cells may play important roles in mediating the detrimental effects of high concentration of sodium. Elevated concentration of salt (sodium chloride) has been found to induce proinflammatory interleukin 17 (IL17)-producing helper $\mathrm{T}\left(\mathrm{T}_{\mathrm{H}} 17\right)$ cells through the $\mathrm{p} 38$ MAPK/nuclear factor of activated T cells 5 (NFAT5) pathway and the serum/glucocorticoid-regulated kinase 1 /forkhead box protein $\mathrm{O} 1$ pathway both in vitro and in vivo $[6,7]$. Consequently, high-salt diet (HSD) exacerbates autoimmune encephalomyelitis in mice $[6,7]$. In addition, hypertension and renal injury induced by HSD in salt-sensitive rats are accompanied by increased infiltration of T cells in kidneys [8]. Similarly, cardiovascular damages induced by HSD in salt-sensitive rats are associated with macrophage infiltration. Conversely, amelioration of these damages is associated with decreased number of macrophages in cardiovascular system $[9,10]$. More recent data show that high salt has direct stimulatory effects on migration of macrophages and expansion of monocyte subpopulation as well as formation of monocyte-platelet aggregates $[11,12]$. However, the impacts of high salt on macrophage activation and inflammation as well as the underlying mechanisms have not been clearly elucidated.

Macrophages are a heterogeneous population of immune cells that serve essential functions in development, homeostasis, tissue repair and immunity [13, 14]. Such heterogeneity is manifested in not only the origins of tissue-resident macrophages but also the wide range of activation states of macrophages [13-17]. Macrophage activation with different transcriptional profiles in response to different stimuli is an important path for macrophages to execute their diverse functions. Recently, it has been recommended to define the activators when describing macrophage activation states [18]. For examples, lipopolysaccharide (LPS) activates M(LPS), interferon- $\gamma$ activates M(IFN- $\gamma$ ), and IL4 activates M(IL4). This paradigm has been put forward to reflect the more complete spectrum of macrophage activation than the paradigm of classical macrophage activation (M1) and alternative macrophage activation (M2) [17-19].

Macrophage activation plays pivotal roles in the initiation and resolution of inflammation under a variety of pathological conditions [13, 14]. For instance, pulmonary macrophages are instrumental in both acute onset and resolution of lung inflammation in both patients with acute respiratory distress syndrome and mouse models of acute lung injury induced by inflammatory stimuli such as LPS $[20,21]$. On one hand, pulmonary macrophages are equipped to initiate a prompt innate immune response through secretion of inflammatory cytokines and recruitment of more immune cells including monocytes and neutrophils. On the other hand, these cells are critical in resolution of lung inflammation and repair by transition from proinflammatory to anti-inflammatory and reparative phenotypes.

In the present study, we set out to explore the direct influence of high salt on macrophage activation and inflammation and to delineate the underlying molecular mechanisms. We first determined the transcriptional and translational expression profiles of high-salt-treated human monocyte-derived macrophages in order to define the activation state. Then we use mouse bone marrow-derived macrophages (BMDMs) to study the effects of high salt alone and in combination with LPS or IL4. We further explore the underlying molecular signaling pathways through which high salt regulates macrophage activation. Finally, we investigate the impacts of high salt on macrophage activation and inflammation in vivo.

\section{Results}

High salt induces a specific activation state, $M(\mathrm{Na})$, in both human and mouse macrophages

To investigate the roles of high salt (increased $\mathrm{NaCl}$ ) in macrophage activation, we treated human monocyte-derived macrophages with additional 17-51 mM $\mathrm{NaCl}$ in the culture medium. RNA-Seq results showed that high salt significantly promoted expression of proinflammatory genes in human macrophages (Figure 1A). The increased expression was also demonstrated by quantitative RT-PCR (qRT-PCR) (Figure 1B). These genes included chemokines such as chemokine (C-C motif) ligand 2 (CCL2), chemokine (C-C motif) ligand 8 (CCL8), chemokine (C-X-C motif) ligand 1 (CXCL1), and chemokine (C-X-C motif) ligand 2 (CXCL2); cytokines such as IL1 beta (IL1B) and IL8; cell surface receptors such as $\mathrm{C}-\mathrm{C}$ chemokine receptor type 2 (CCR2), tolllike receptor 3 (TLR3), toll-like receptor 4 (TLR4), and cluster of differentiation 14 (CD14). High salt enhanced expression of proinflammatory genes such as $C C L 8$, CXCL1, IL8, and CCR2 in a dose-dependent manner in human macrophages (Supplementary information, Figure S1A). Furthermore, ELISA results showed that protein levels of CXCL1 and IL8 were also increased by highsalt treatment (Figure 1C). In contrast, high salt markedly suppressed gene expression of anti-inflammatory and proendocytic molecules as demonstrated by RNA- 
A

\section{Pro-inflammation}

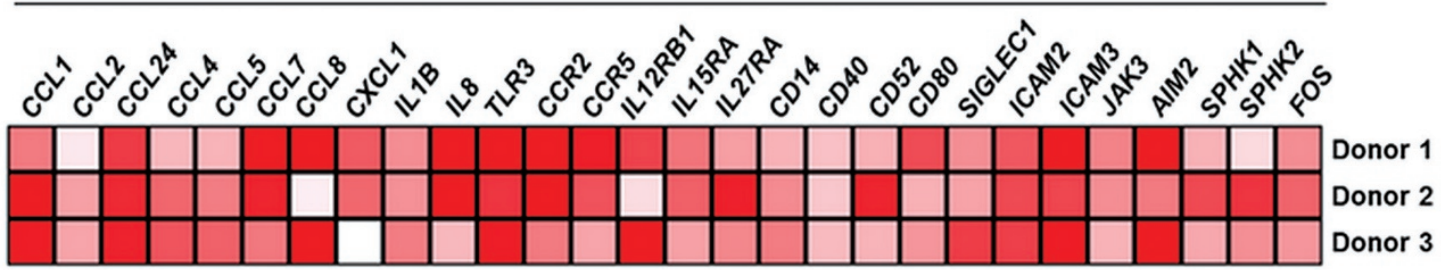

Expression ( $\log _{2}$ fold)

0

2

B

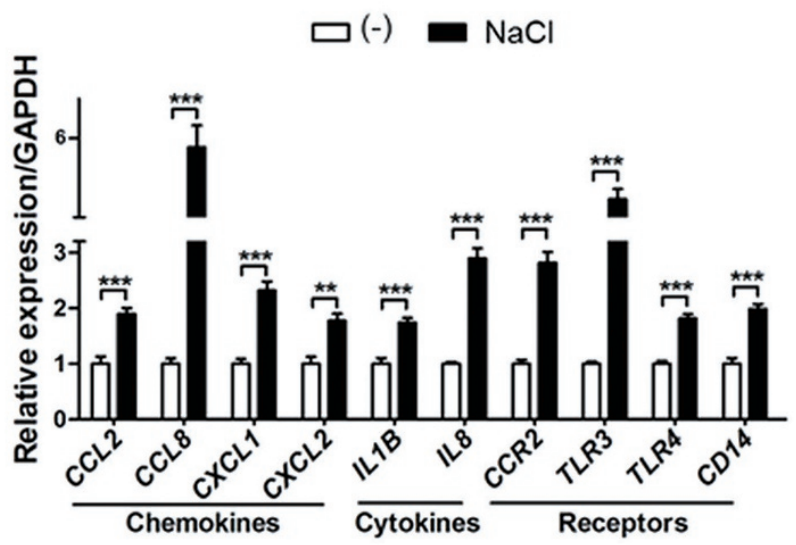

C

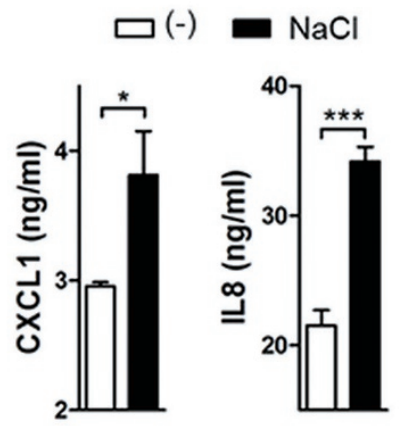

D

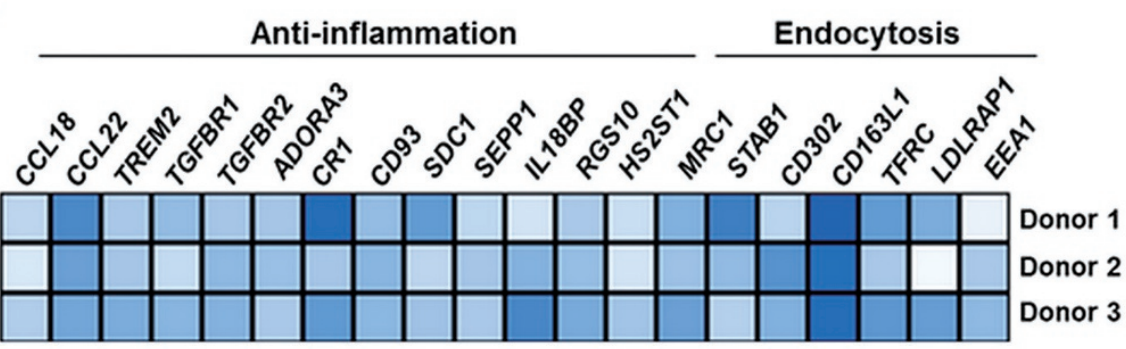

Expression ( $\log _{2}$ fold)

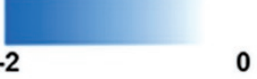

E

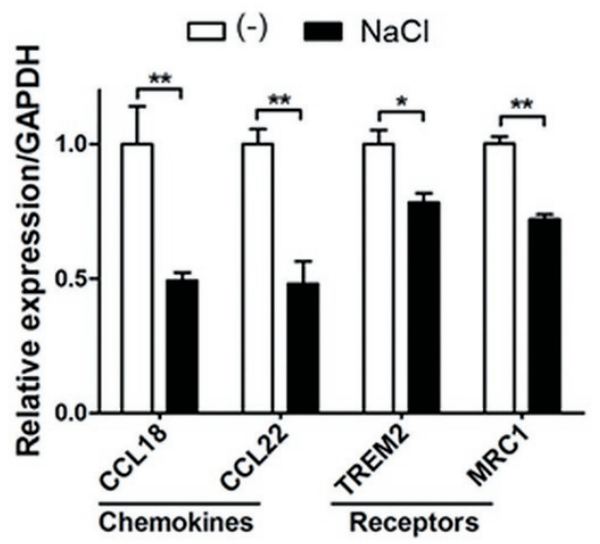

F

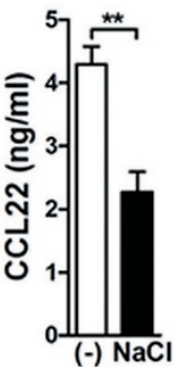

G

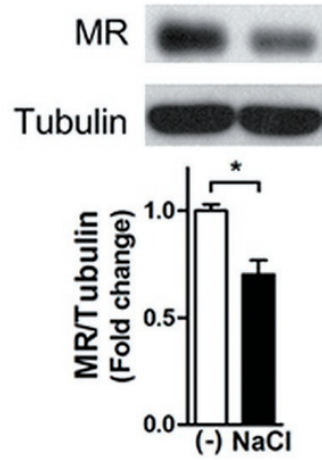


Seq (Figure 1D). Results of qRT-PCR further showed that mRNA levels of chemokine (C-C motif) ligand 18 (CCL18) and chemokine (C-C motif) ligand 22 (CCL22) fell by half in high-salt-treated human macrophages compared with those in untreated cells (Figure 1E). Gene expression levels of triggering receptor expressed on myeloid cells 2 (TREM2) and mannose receptor, $\mathrm{C}$ type 1 (MRC1, also known as $M R$ ) were also significantly reduced by high salt (Figure 1E). Notably, high salt suppressed gene expression of CCL22 and MRC1 dose-dependently (Supplementary information, Figure S1A). High salt also reduced protein levels of CCL22 (Figure $1 \mathrm{~F}$ ) and MR (Figure $1 \mathrm{G}$ ) as determined by ELISA and western blotting, respectively. Similarly, in mouse BMDMs, additional $51 \mathrm{mM} \mathrm{NaCl}$ significantly increased expression level of proinflammatory genes (Supplementary information, Figure S1B) and decreased that of anti-inflammatory and proendocytic genes (Supplementary information, Figure S1C). Notably, additional 51 $\mathrm{mM} \mathrm{NaCl}$ treatment did not induce apoptosis of mouse BMDMs (Supplementary information, Figure S1D).

Gene ontology analysis of RNA-Seq results showed that high salt affected a variety of molecular functions such as protein binding and nucleic acid binding in human macrophages (Supplementary information, Figure S1E and S1F). Taken together, these data suggest that high salt induces a specific macrophage activation state and we term it $\mathrm{M}(\mathrm{Na})$. These macrophages mainly show the characteristics of enhanced proinflammatory gene expression and suppressed anti-inflammatory gene expression.

\section{High salt potentiates M(LPS) and suppresses M(IL4)}

The next interesting question is whether high salt affects the phenotypes of M(LPS) and M(IL4) that are, respectively, classical proinflammatory and anti-inflammatory macrophage activation state [18]. To address this question, we treated mouse BMDMs with additional $\mathrm{NaCl}$ or in combination with LPS or IL4 and measured gene expression by qRT-PCR and protein expression by western blotting. High salt time-dependently enhanced
LPS-induced expression of proinflammatory genes including CXCL1, CXCL2, IL12 alpha (IL12A), IL12 beta (IL12B), Cyclooxygenase 2 (COX2), and inducible nitric oxide synthase (iNOS) in mouse BMDMs (Figure 2A). Consistent with the results of gene expression, protein levels of COX2 and iNOS were also much higher in macrophages treated by high salt and LPS together than LPS alone (Figure 2B). Conversely, high salt strongly inhibited IL4-induced expression of genes including found in inflammatory zone 1 (Fizzl), mouse macrophage galactose $\mathrm{N}$-acetylgalactosamine specific lectin 1 (mMGL1), mouse macrophage galactose $\mathrm{N}$-acetylgalactosamine specific lectin 2 ( $m M G L 2)$, macrophage mannose receptor $(M M R)$, chitinase-like 3 (Chil3, also known as YM1), and interferon regulatory factor 4 (IRF4) (Figure 2C), as well as protein expression of mMGL2, MMR, YM1 and IRF4 (Figure 2D). In addition, the potentiation of M(LPS) by high salt was observed in human macrophages. High salt significantly promoted gene expression of proinflammatory molecules (Supplementary information, Figure S2A) and protein expression of CXCL1 and IL8 (Supplementary information, Figure S2B) in human macrophages stimulated with LPS. We did not detect any LPS in the $\mathrm{NaCl}$ we used (Supplementary information, Figure S2C), confirming that the effects of $\mathrm{NaCl}$ were not due to LPS contamination. In addition, LPS increased expression levels of anti-inflammatory genes such as activating transcription factor 3 (ATF3), IL10, IL1 receptor antagonist (IL1RA), suppressor of cytokine signaling 1 (SOCS1), and nuclear factor of kappa light polypeptide gene enhancer in B-cells inhibitor, alpha (IKBA) $24 \mathrm{~h}$ after the treatment. In contrast, high salt either deceased or did not significantly change expression levels of these genes (Supplementary information, Figure S2D).

p38 mediates the upregulation of proinflammatory genes of $M(N a)$ and high-salt-induced potentiation of $M(L P S)$

The effects of high salt are probably due to the elevation of sodium, chloride, or extracellular osmolarity. To test these possibilities, we treated BMDMs with addi-

Figure 1 High salt induces a specific activation state in human macrophages. Human monocyte-derived macrophages were treated with additional $51 \mathrm{mM} \mathrm{NaCl}$ for $24 \mathrm{~h}$. (A) High salt promotes gene expression of proinflammatory molecules as determined by RNA-Seq. mRNA levels of high-salt-treated human macrophages are presented as fold changes relative to those of untreated human macrophages. (B) High salt promotes gene expression of proinflammatory molecules as determined by qRT-PCR. (C) High salt promotes protein expression of proinflammatory molecules. Protein levels of CXCL1 and IL8 in conditioned media from human macrophages were quantified using ELISA. (D) High salt inhibits gene expression of anti-inflammatory and proendocytic molecules as determined by RNA-Seq. (E) High salt inhibits gene expression of anti-inflammatory and proendocytic molecules as determined by GRT-PCR. (F) High salt decreases protein level of CCL22 in conditioned media from human macrophages as determined by ELISA. (G) High salt decreases protein level of MR in human macrophages as determined by western blotting. Tubulin was used as a loading control. Representative results of qRT-PCR, ELISA and western blotting from three independent experiments are shown. ${ }^{*} P<0.05 ;{ }^{* \star} P<0.01$; ${ }^{* \star} P<0.001$. 
A
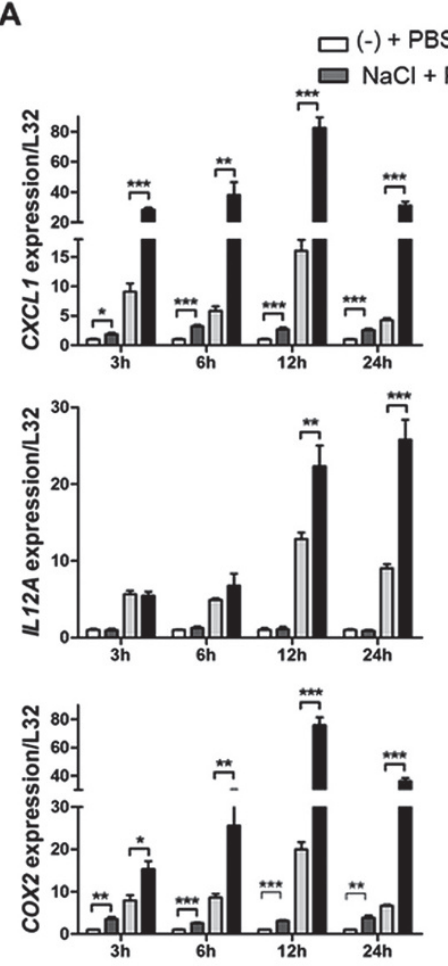

C
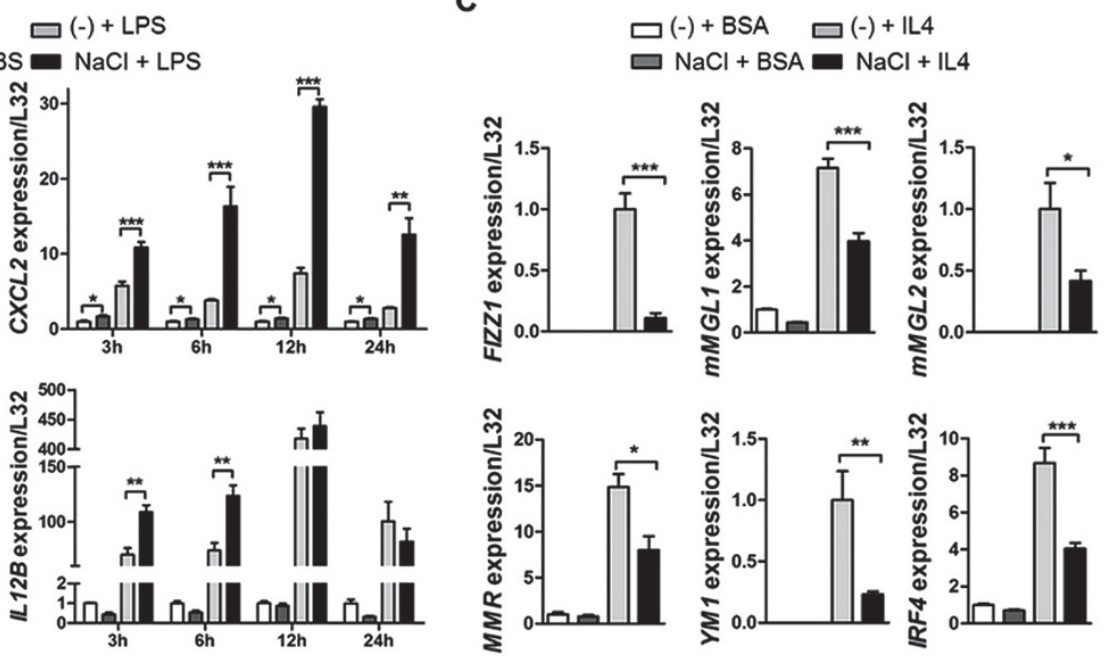

\section{B}

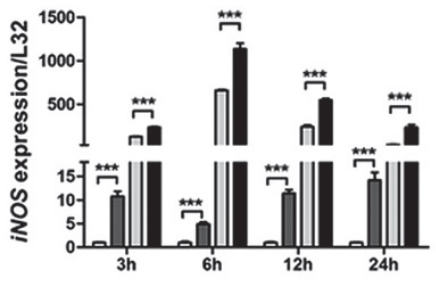

D
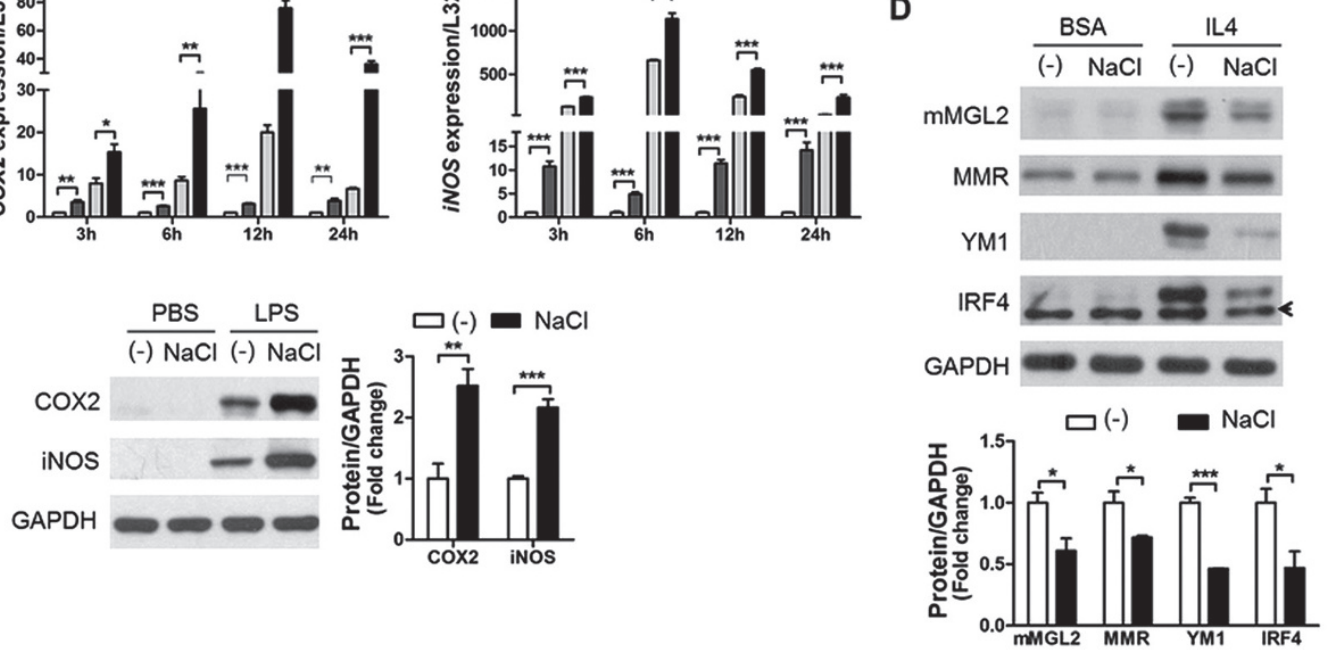

Figure 2 High salt potentiates M(LPS) and suppresses M(IL4) in mouse BMDMs. (A) High salt enhances LPS-induced gene expression. BMDMs were pretreated with or without additional $51 \mathrm{mM} \mathrm{NaCl}$ for $12 \mathrm{~h}$, and then treated with or without $100 \mathrm{ng} /$ $\mathrm{ml}$ LPS in the presence or absence of additional $51 \mathrm{mM} \mathrm{NaCl}$ for the indicated time periods. (B) High salt enhances LPS-induced protein expression. BMDMs were treated with or without additional $51 \mathrm{mM} \mathrm{NaCl}$ for $24 \mathrm{~h}$ and $100 \mathrm{ng} / \mathrm{ml} \mathrm{LPS}$ or PBS was added during the latter half of the treatment. Protein expression of COX2 and iNOS was measured and GAPDH was used as a loading control. (C) High salt suppresses IL4-induced gene expression. BMDMs were treated with $10 \mathrm{ng} / \mathrm{ml}$ IL4 or $0.1 \%$ BSA in the presence or absence of additional $51 \mathrm{mM} \mathrm{NaCl}$ for $24 \mathrm{~h}$. (D) High salt suppresses IL4-induced protein expression. BMDMs were treated in the same way as in C. Arrowhead: non-specific bands. For all panels, gene expression was measured using qRT-PCR and protein expression was measured using western blotting. ${ }^{*} P<0.05 ;{ }^{* \star} P<0.01 ;{ }^{* * \star} P<0.001$.

tional $51 \mathrm{mM} \mathrm{NaGluconate}$ or $102 \mathrm{mM}$ sucrose with the same osmolarity. The results showed that NaGluconate treatment significantly induced proinflammatory gene expression, while sucrose did not (Supplementary information, Figure S3A). These results suggest that increase of sodium concentration was necessary for high salt to induce $\mathrm{M}(\mathrm{Na})$ while chloride was dispensable, and that osmolarity elevation alone was not enough to promote proinflammatory gene expression.
Then, what are the intracellular mechanisms that mediate the effects of high salt? p38 mitogen-activated protein kinase (MAPK) plays important roles in cellular response to stress stimuli including cytokines and heat shock [22]. Recent reports have shown that $\mathrm{p} 38$ is strikingly activated by high salt in $\mathrm{T}_{\mathrm{H}} 17$ cells $[6,7]$. We therefore explored the possibility that $\mathrm{p} 38$ also mediated the effects of high salt in macrophages. As expected, phosphorylation of p38 was significantly increased in BMDMs treated with 
additional $51 \mathrm{mM} \mathrm{NaCl}$ (Figure 3A). When SB203580 (SB), a selective inhibitor of $\mathrm{p} 38 \alpha$ and $\mathrm{p} 38 \beta$, was added, the induction of gene expression of $C X C L 2, I L 1 B, C D 14$, and $C O X 2$ by high salt was significantly alleviated. As a result, gene expression levels of these proinflammatory molecules were decreased in SB group compared with dimethyl sulphoxide (DMSO) group of high-salt-treated BMDMs (Figure 3B). Furthermore, we used BMDMs from myeloid-specific p38 $\alpha$-knockout mice and floxed control littermates (Supplementary information, Figure S3B) to test the involvement of $\mathrm{p} 38$. Deficiency of $\mathrm{p} 38 \alpha$ significantly inhibited high-salt-induced $C O X 2$ gene expression in BMDMs (Supplementary information, Figure $\mathrm{S} 3 \mathrm{C}$ ). However, the decrease in gene expression levels of $m M G L 1$ and $M M R$ induced by high salt was independent of p38 (Supplementary information, Figure S3D).

We next investigated whether p38 mediated the effects of high salt on M(LPS). High salt further increased phosphorylation of p38 in BMDMs treated by LPS for 1 h (Figure 3C), but not for 3 or 6 h (Supplementary information, Figure S3E). Under LPS stimulation SB also significantly inhibited high-salt-enhanced gene expression of proinflammatory molecules classically induced by LPS, some of which (e.g., CXCL2, IL1B and IL12A) were completely blocked (Figure 3D). Consistently, high-saltinduced increase in protein levels of COX2 and iNOS was also markedly alleviated by SB treatment in mouse BMDMs under LPS stimulation (Figure 3E). Similarly, high-salt-induced increase of gene expression of $C X C L 1$, CXCL2, COX2, and $i N O S$ was significantly smaller in LPS-treated BMDMs from p38 $\alpha$-knockout mice than floxed control mice (Supplementary information, Figure S3F). However, SB did not lessen high-salt-induced suppression on gene expression of FIZZ1, MMR, YM1, or IRF4 in BMDMs treated with IL4 (Supplementary information, Figure S3G). These results together demonstrated that p38 mediated high-salt-induced proinflammation in macrophages and its potentiation of M(LPS).

Erk1/2 mediates the gene expression of $M(\mathrm{Na})$ as well as high-salt-induced potentiation of M(LPS) and suppression of M(IL4)

Besides p38, Extracellular signal-regulated kinase1/2 (Erk1/2) and c-Jun N-terminal kinase (JNK) are two other major subfamilies of MAPKs that play critical roles in transducing intracellular signaling when cells respond to stress stimuli [22]. We continued to investigate whether Erk1/2 and JNK also mediated the effects of high salt.

First, we measured the levels of phosphorylated and total Erk1/2 and JNK in BMDMs treated with or without additional $51 \mathrm{mM} \mathrm{NaCl}$. The results showed that high salt significantly increased Erk1/2 phosphorylation but did not affect the level of total Erk1/2 (Figure 4A). In addition, high salt did not affect either phosphorylated or total level of JNK (Supplementary information, Figure S4A). Therefore, we focused on studying the roles of Erk1/2 using a selective MAPK kinase (MEK) inhibitor PD98059 (PD) to inhibit Erk1/2 activation and macrophages from Erk1-knockout mice. PD markedly attenuated high-salt-induced increase of CCL8,CD14,COX2 and $i N O S$ gene expression (Figure 4B) as well as decrease of $A T F 3, I R F 4, m M G L 1$ and $M M R$ gene expression (Figure 4C). We used BMDMs from Erkl-knockout and wildtype mice (Supplementary information, Figure S4B) to further test the involvement of Erk1/2. Erk1 deficiency alleviated high-salt-induced upregulation of proinflammatory genes such as CCL8, COX2 and iNOS (Supplementary information, Figure S4C) and downregulation of $M M R$ (Supplementary information, Figure S4D) in BMDMs.

We next tested the possibility that high-salt-induced potentiation of M(LPS) depends on Erk1/2 activation. High salt further enhanced Erk1/2 activation in LPS-treated BMDMs (Figure 4D). Moreover, under the combined stimulation of LPS and high salt, PD still significantly inhibited proinflammatory gene expression (Figure 4E). Consistently, COX2 and iNOS protein levels were significantly lower in BMDMs treated with PD than those with DMSO (Figure 4F).

In addition, we noted that high salt increased p-Erk $1 / 2$ level in IL4-treated BMDMs, while IL4 did not affect p-Erk1/2 by itself (Figure $4 \mathrm{G}$ ). Therefore, we further tested whether Erk1/2 mediated the suppression of M(IL4) by high salt. PD completely abolished or significantly lessened high-salt-induced decrease in gene expression of FIZZ1, MMR, YM1, and IRF4 (Figure 4H) as well as protein expression of MMR, YM1, and IRF4 (Figure 4I). These results indicated that the increase of Erk $1 / 2$ phosphorylation was necessary for high salt to suppress M(IL4). Putting these results together, we demonstrated that Erk1/2 mediated $\mathrm{M}(\mathrm{Na})$ activation and high-salt-induced potentiation of M(LPS) and suppression of M(IL4).

cFos/AP1 mediates the proinflammatory gene expression of $M(\mathrm{Na})$ and high-salt-induced potentiation of $M(L P S)$

Activator protein 1 (AP1) is a transcription factor that predominantly exists as a heterodimer of cFos and cJun and is critical for response to inflammatory stimuli [15]. Moreover, both p38 and Erk1/2 can phosphorylate cFos $[23,24]$, enhancing transcriptional activity of AP1 and ultimately promoting expression of its target genes. We therefore propose that high salt enhances cFos phosphorylation and transcriptional activity of AP1 through p38 and/or Erk1/2 activation, leading to the induction of 
A
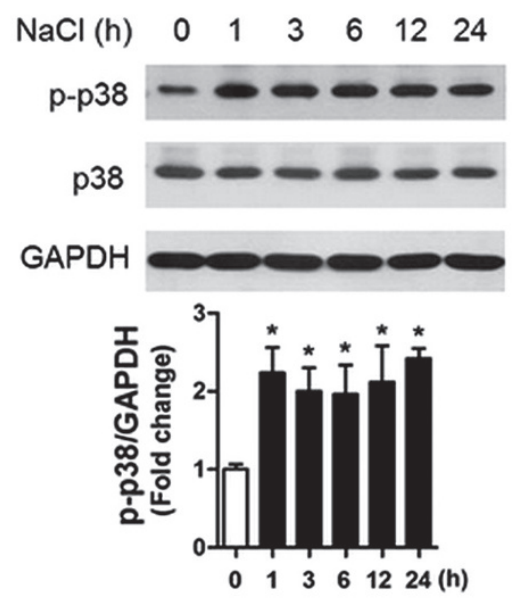

C

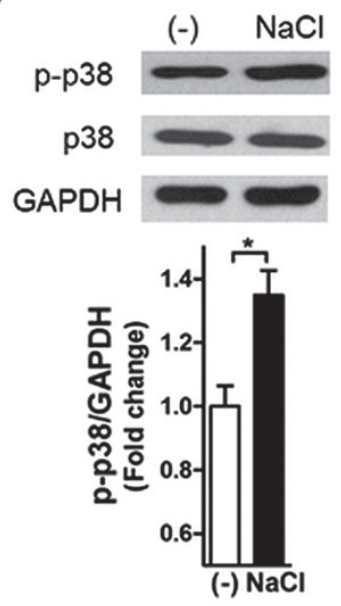

B
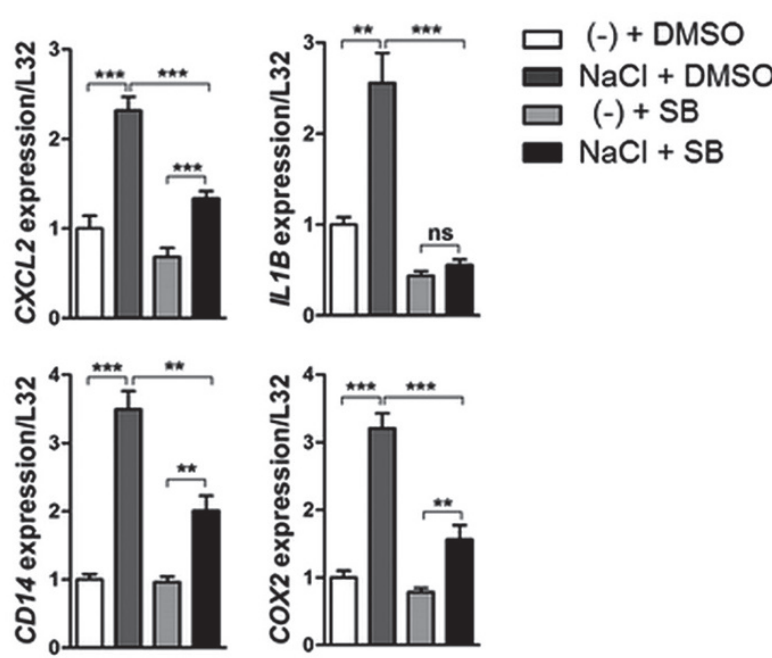

$(-)$

D

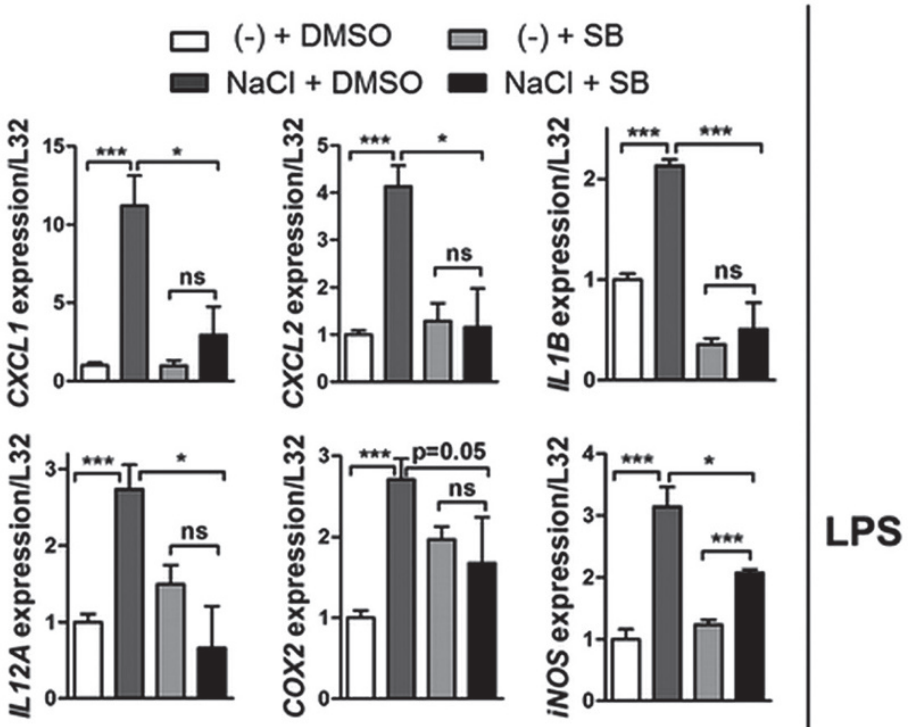

$\mathbf{E}$
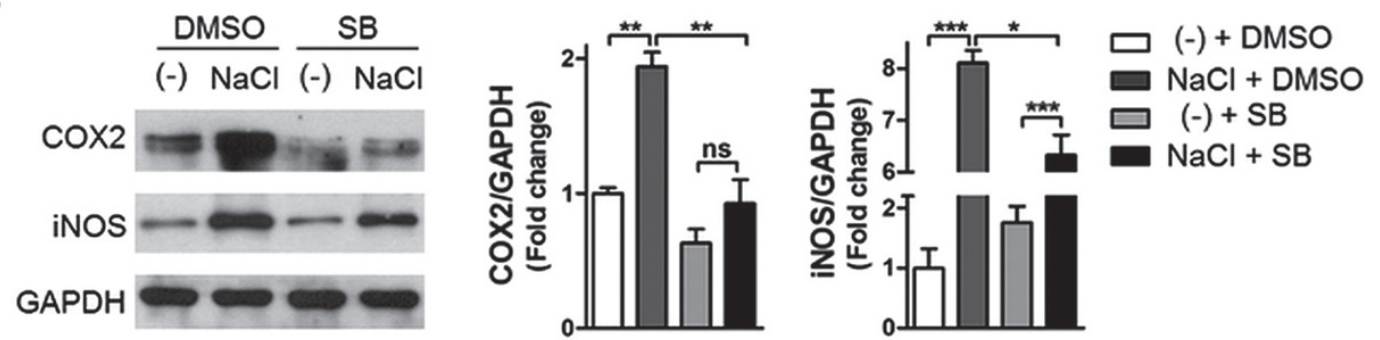

Figure 3 p38 MAPK mediates the upregulation of proinflammatory genes of $\mathrm{M}(\mathrm{Na})$ and high-salt-induced potentiation of M(LPS). (A) High salt activates p38 in macrophages. BMDMs were treated with or without additional $51 \mathrm{mM} \mathrm{NaCl}$ for the indicated time periods. p-p38 and total p38 levels were measured and GAPDH was used as a loading control. (B) SB inhibits high-salt-promoted expression of proinflammatory genes. BMDMs were pretreated with $10 \mu \mathrm{M} \mathrm{SB}$ or DMSO for $2 \mathrm{~h}$ and then treated with or without additional $51 \mathrm{mM} \mathrm{NaCl}$ for $24 \mathrm{~h}$ in the presence of $5 \mu \mathrm{M}$ SB or DMSO. (C) High salt enhances LPS-induced p38 phosphorylation. BMDMs were treated with or without additional $51 \mathrm{mM} \mathrm{NaCl}$ for $13 \mathrm{~h}$ and $100 \mathrm{ng} / \mathrm{ml} \mathrm{LPS}$ was added during the last $1 \mathrm{~h}$ of the treatment. (D, E) p38 mediates high-salt-enhanced response to LPS. BMDMs were treated by the same procedure as in B, except that $100 \mathrm{ng} / \mathrm{ml}$ LPS was added to all groups during the last $12 \mathrm{~h}$ of the treatment. Expression of the indicated genes (D) and proteins (E) was quantified. For all panels, gene expression was measured using qRT-PCR and protein expression or phosphorylation was measured using western blotting. ns, not significant; ${ }^{*} P<0.05$; ${ }^{* *} P$ $<0.01 ;{ }^{* * *} P<0.001$. 
A
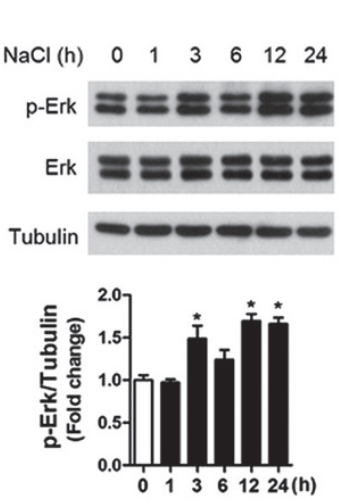

D

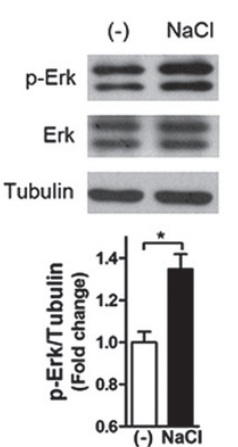

G
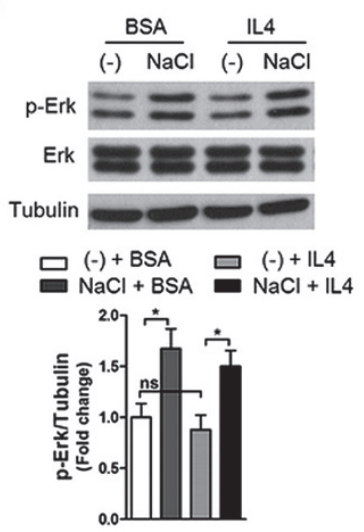

B

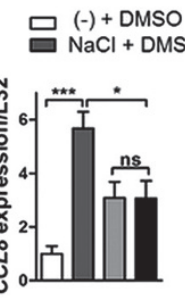

E
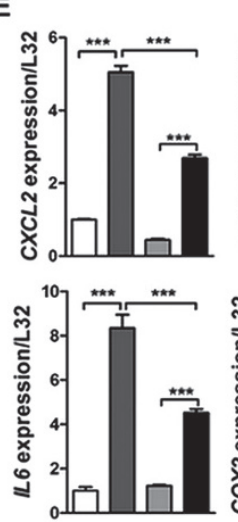

H

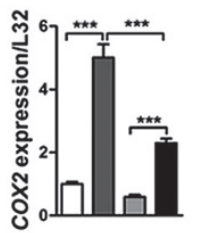

C

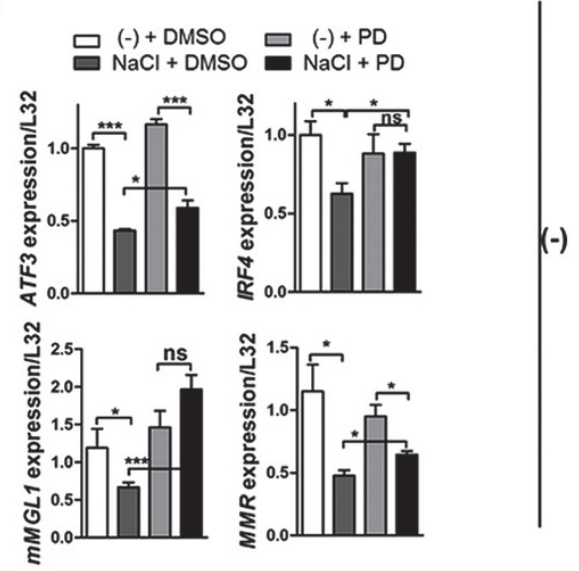

$\mathbf{F}$

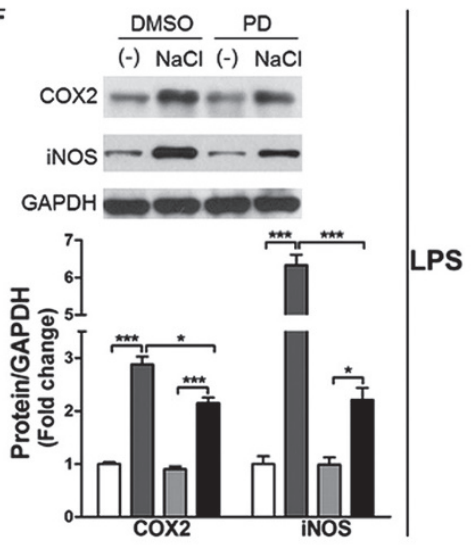

I
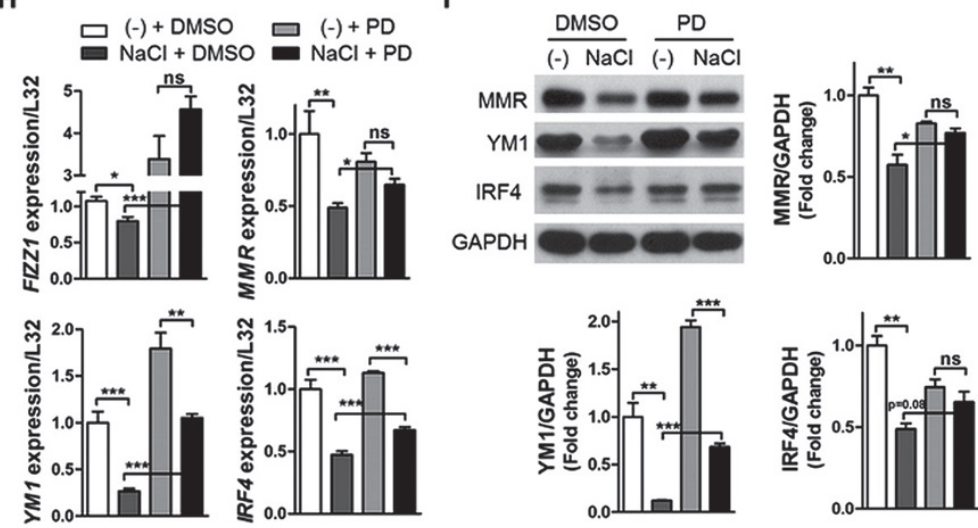

IL4

Figure 4 Erk1/2 MAPK mediates the gene expression of $\mathrm{M}(\mathrm{Na})$ as well as high-salt-induced potentiation of $\mathrm{M}(\mathrm{LPS})$ and suppression of M(IL4). (A) High salt activates Erk1/2 in macrophages. BMDMs were treated with or without additional $51 \mathrm{mM} \mathrm{NaCl}$ for the indicated time periods. p-Erk and total Erk1/2 (Erk) levels were measured and tubulin was used as a loading control. (B, C) PD inhibits high-salt-induced upregulation of proinflammatory gene expression (B) and downregulation of anti-inflammatory and proendocytic gene expression (C). BMDMs were pretreated with $20 \mu \mathrm{M}$ PD or DMSO for $1 \mathrm{~h}$ and then treated with or without additional $51 \mathrm{mM} \mathrm{NaCl}$ for $24 \mathrm{~h}$ in the continued presence of $20 \mu \mathrm{M}$ PD or DMSO. (D) High salt enhances LPS-induced Erk1/2 phosphorylation. BMDMs were treated with or without additional $51 \mathrm{mM} \mathrm{NaCl}$ for $13 \mathrm{~h}$ and $100 \mathrm{ng} / \mathrm{ml}$ LPS was added during the last $1 \mathrm{~h}$ of the treatment. (E, F) Erk1/2 mediates high-salt-induced potentiation of M(LPS). BMDMs were treated by the same procedure as in $\mathbf{B}$, except that $100 \mathrm{ng} / \mathrm{ml}$ LPS was added to all groups during the last $12 \mathrm{~h}$. Expression of the indicated genes (E) and proteins (F) was quantified. (G) High salt increases p-Erk1/2 at baseline and under IL4 treatment. BMDMs were treated with $10 \mathrm{ng} / \mathrm{ml} \mathrm{IL4}$ or BSA in the presence or absence of additional $51 \mathrm{mM} \mathrm{NaCl}$ for $6 \mathrm{~h}$. (H, I) Erk1/2 mediates high-salt-induced suppression of M(IL4). BMDMs were treated in the same way as in C, except that $10 \mathrm{ng} / \mathrm{ml}$ IL4 was added to all groups during the last $24 \mathrm{~h}$. Expression of the indicated genes $(\mathbf{H})$ and proteins $(\mathbf{I})$ was quantified. For all panels, gene expression was measured using qRT-PCR and protein expression or phosphorylation was measured using western blotting. ns, not significant; ${ }^{*}<0.05$; ${ }^{* *} P<0.01 ;{ }^{* * *} P<0.001$. 
$\mathrm{M}(\mathrm{Na})$ and potentiation of $\mathrm{M}(\mathrm{LPS})$.

As expected, cFos phosphorylation (p-cFos) was increased by high salt in BMDMs time-dependently but the level of total cFos was not affected (Figure 5A). The high-salt-induced upregulation of proinflammatory genes (CCL8, CXCL1, CXCL2, IL1B, COX2, and iNOS) was markedly inhibited by SR11302 (SR), a selective inhibitor of AP1 (Figure 5B). Deficiency of cFos (Supplementary information, Figure S5A) had the similar inhibitory effects in BMDMs (Supplementary information, Figure S5B). Interestingly, both SR treatment and cFos deficiency in macrophages completely blocked high-salt-induced upregulation of $C X C L 2$, indicating that the effect of high salt on this gene was exclusively dependent on cFos. Furthermore, both SB and PD dramatically attenuated high-saltinduced p-cFos, indicating that the effects of high salt on p-cFos were mediated by p-p38 (Figure 5C) and p-Erk1/2 (Figure 5D). Interestingly, PD also decreased p-cFos in BMDMs without high-salt treatment (Figure 5D).

The level of phosphorylated cJun or total cJun was not increased by high salt (Supplementary information, Figure S5C), consistent with the unchanged activation of JNK upon high-salt treatment (Supplementary information, Figure S4A). In addition, high-salt treatment did not affect the level of phosphorylated P65 (p-P65), phosphorylated I $\kappa \mathrm{B} \alpha(\mathrm{p}-\mathrm{I} \kappa \mathrm{B} \alpha)$, total I $\mathrm{KB} \alpha$ (Supplementary information, Figure S5D), or IRF5 (Supplementary information, Figure S5E). These results suggest that activation of IRF5 or nuclear factor kappa B (NFkB) was not affected by high salt.

Under LPS stimulation, high-salt treatment further enhanced p-cFos in BMDMs (Figure 5E). Consistently, SR significantly weakened high-salt-induced potentiation of M(LPS). As a result, expression levels of proinflammatory genes (CXCL1, CXCL2, IL1B,IL6, COX2, and $i N O S$ ) were much lower in SR-treated groups than DMSO-treated groups in BMDMs treated by additional $51 \mathrm{mM} \mathrm{NaCl}$ and LPS together (Figure 5F). Protein expression levels of COX2 and iNOS were also lower in SR-treated groups (Figure 5G). Moreover, under LPS co-stimulation, highsalt-induced enhancement of p-cFos was completely abolished by SB (Figure 5H) or p38 $\alpha$ deficiency (Supplementary information, Figure S5F). SB also decreased p-cFos in BMDMs treated with LPS in the absence of high salt (Figure 5H). However, PD did not affect highsalt-induced upregulation of $\mathrm{p}-\mathrm{cF}$ os level in BMDMs treated with high salt and LPS together (Supplementary information, Figure S5G).

Taken together, these data showed that cFos/AP1 mediated the proinflammatory gene expression of $\mathrm{M}(\mathrm{Na})$ downstream of both p-p38 and p-Erk1/2. In addition, cFos/AP1 mediated-high-salt-induced potentiation of M(LPS) depends on p-p38, but is independent of p-Erk1/2 in mouse BMDMs.

STAT6 mediates the downregulation of anti-inflammatory and proendocytic gene expression of $\mathrm{M}(\mathrm{Na})$ as well as high-salt-induced suppression of M(IL4)

Signal transducer and activator of transcription 6 (STAT6) is a classical transcription factor that regulates anti-inflammatory and proendocytic gene expression in macrophages $[15,16]$. Thereby, we tested whether STAT6 mediated the effects of high salt. High salt time-dependently decreased the protein level of phosphorylated STAT6 (p-STAT6) in BMDMs (Figure 6A). Unexpectedly, total STAT6 protein level was also decreased (Figure 6A) while the mRNA level of STAT6 was not (Supplementary information, Figure S6A). To further examine whether the downregulation of STAT6 accounted for the inhibitory effects of high salt in macrophages, we stably overexpressed murine STAT6 in mouse macrophage cell line RAW264.7. Overexpression of STAT6 significantly attenuated high-salt-induced decrease in expression of genes including Kruppel-like factor 4 (KLF4), peroxisome proliferator-activated receptor gamma (PPARg), and MMR (Figure 6B). Given that p-Erk1/2 mediated the suppression of anti-inflammatory and proendocytic gene expression of $\mathrm{M}(\mathrm{Na})$, we next investigated whether $\mathrm{p}$-Erk1/2 mediated the high-saltinduced downregulation of p-STAT6 and total STAT6. When PD was added, downregulation of both p-STAT6 and total STAT6 was completely abrogated in BMDMs (Figure 6C).

In the presence of IL4, additional $51 \mathrm{mM} \mathrm{NaCl}$ treatment also decreased the levels of p-STAT6 and total STAT6 in BMDMs (Figure 6D). Consistently, high-saltinduced suppression of protein levels of MMR and IRF4, two classical markers of M(IL4), was alleviated by STAT6 overexpression in RAW264.7 cells. As a result, RAW264.7 cells with STAT6 overexpression had much higher expression of MMR and IRF4 than those with GFP overexpression when treated with high salt and IL4 together (Figure $6 \mathrm{E})$. These data indicated that the attenuation of STAT6 contributed to high-salt-induced suppression of M(IL4). Furthermore, this attenuation was abolished by PD in BMDMs (Figure $6 \mathrm{~F}$ ), suggesting that $\mathrm{p}$-Erk1/2 mediated such attenuation in the presence of IL4. STAT6 mRNA level was not affected by high salt in IL4-treated BMDMs (Supplementary information, Figure S6B). These results together showed that high salt attenuated STAT6 through promoting Erk1/2 phosphorylation, ultimately inhibiting the anti-inflammatory and proendocytic gene expression of $\mathrm{M}(\mathrm{Na})$ as well as suppressing M(IL4).

Summarizing all the results regarding high-salt-in- 
duced intracellular signaling pathways, we propose a model of the mechanisms (Figure 6G). In this model, high salt induces the increase of proinflammatory gene and protein expression of $\mathrm{M}(\mathrm{Na})$ and $\mathrm{M}(\mathrm{LPS})$ through p38/cFos/AP1 pathway. The proinflammatory profile of $\mathrm{M}(\mathrm{Na})$ is also dependent on Erk1/2/cFos/AP1 signaling. On the other hand, Erk1/2/STAT6 pathway mediates high-salt-induced attenuation of anti-inflammatory and proendocytic gene expression of $\mathrm{M}(\mathrm{Na})$ as well as the suppression of M(IL4).

\section{High salt aggravates lung inflammation}

Our data demonstrated that high salt remarkably promoted gene and protein expression of proinflammatory molecules in both human and mouse macrophages in the absence or presence of LPS. We then asked whether high salt would affect phenotypes of monocytes/macrophages and inflammation in vivo.

We first studied the effects of high salt intake in mice at baseline. Mice were fed with chow diet (CD) or HSD. Alveolar macrophages were collected for gene expression
A

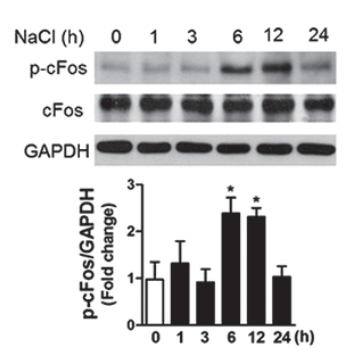

E
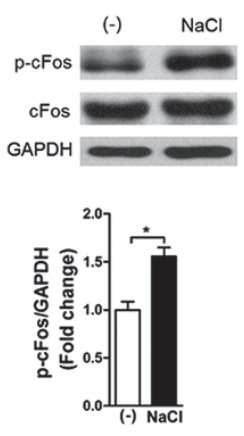

B

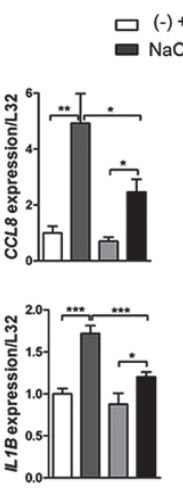

口 (-)+ DMSO $\square(-)+$ SR

$\mathrm{NaCl}+\mathrm{DMSO}=\mathrm{NaCl}+\mathrm{SR}$
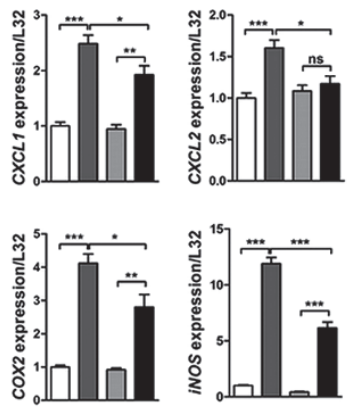

$\mathbf{F}$

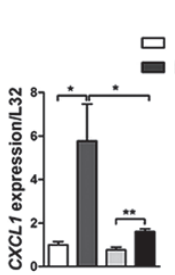

(-) + DMSO 口(-)+SR
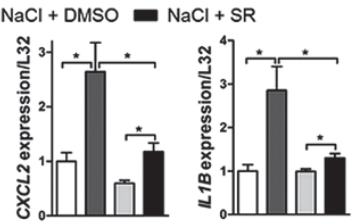

G

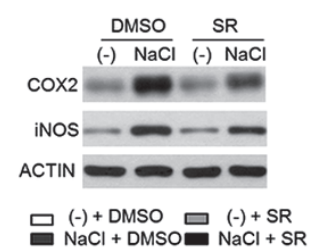

C

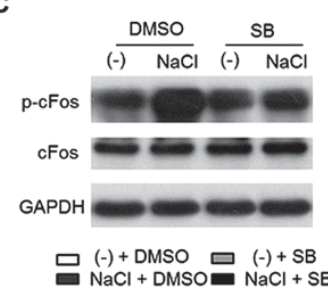

D

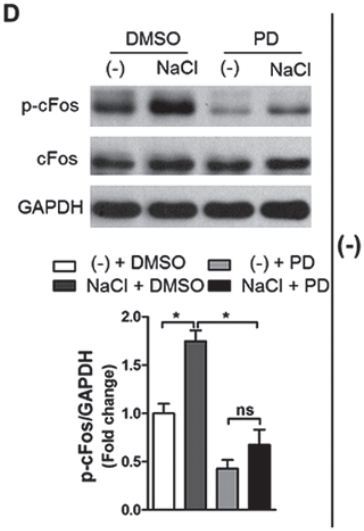

H

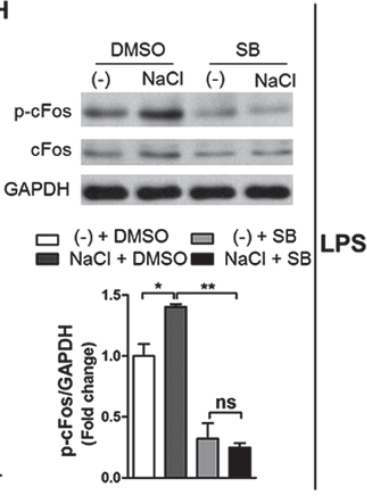

Figure 5 AP1 mediates the proinflammatory gene expression of $M(\mathrm{Na})$ and high-salt-induced potentiation of $M(L P S)$. (A) High salt promotes cFos phosphorylation in macrophages. BMDMs were treated with or without additional $51 \mathrm{mM} \mathrm{NaCl}$ for the indicated time periods. p-cFos and total cFos (cFos) levels were measured and GAPDH was used as a loading control. (B) SR inhibits high-salt-promoted proinflammatory gene expression. BMDMs were pretreated with $10 \mu \mathrm{M}$ SR or DMSO for 1 $\mathrm{h}$ and then treated with or without additional $51 \mathrm{mM} \mathrm{NaCl}$ for $24 \mathrm{~h}$ in the continued presence of $10 \mu \mathrm{M} \mathrm{SR}$ or DMSO. (C) High salt-induced increase of p-cFos depends on p38 activation. BMDMs were pretreated with $10 \mu \mathrm{M}$ SB for $2 \mathrm{~h}$ and then treated with or without additional $51 \mathrm{mM} \mathrm{NaCl}$ for $12 \mathrm{~h}$ in the presence of $5 \mu \mathrm{M}$ SB or DMSO. (D) High salt-induced increase of cFos phosphorylation depends on Erk1/2 activation. BMDMs were pretreated with $20 \mu \mathrm{M} \mathrm{PD}$ for $1 \mathrm{~h}$ and then treated with or without additional $51 \mathrm{mM} \mathrm{NaCl}$ for $12 \mathrm{~h}$ in the presence of $20 \mu \mathrm{M}$ PD or DMSO. (E) High salt enhances LPS-induced p-cFos. BMDMs were treated with or without additional $51 \mathrm{mM} \mathrm{NaCl}$ for $13 \mathrm{~h}$ and $100 \mathrm{ng} / \mathrm{ml}$ LPS was added during the last $1 \mathrm{~h}$ of the treatment. (F, G) AP1 mediates high-salt-enhanced response to LPS. BMDMs were treated by the same procedure as in $\mathbf{B}$, except that $100 \mathrm{ng} / \mathrm{ml}$ LPS was added to all groups during the last $12 \mathrm{~h}$ of the treatment. Expression of the indicated genes (F) and proteins (G) was quantified. (H) High salt-induced increase of p-cFos in the presence of LPS depends on p38 activation. BMDMs were treated by the same procedure as in C, except that $100 \mathrm{ng} / \mathrm{ml}$ LPS was added to all groups during the last $1 \mathrm{~h}$ of the treatment. For all panels, gene expression was measured using qRT-PCR and protein expression or phosphorylation was measured using western blotting. ns, not significant; ${ }^{*} P<0.05 ;{ }^{* *} P<0.01$; ${ }^{* *} P<0.001$. 
A
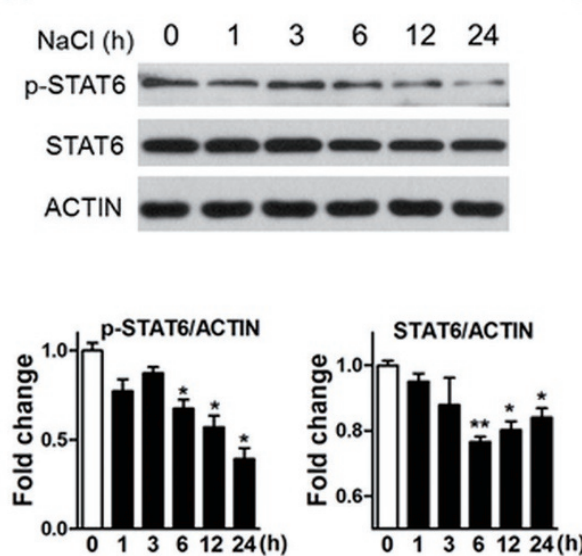

D

(-) $\mathrm{NaCl}$

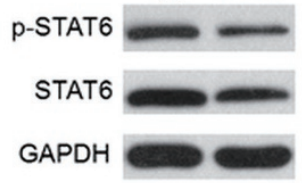

$\square(-)=\mathrm{NaCl}$

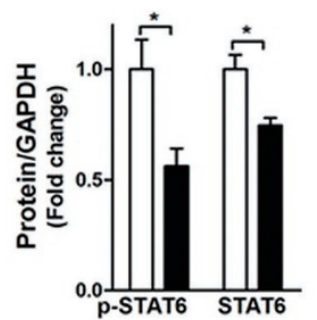

G

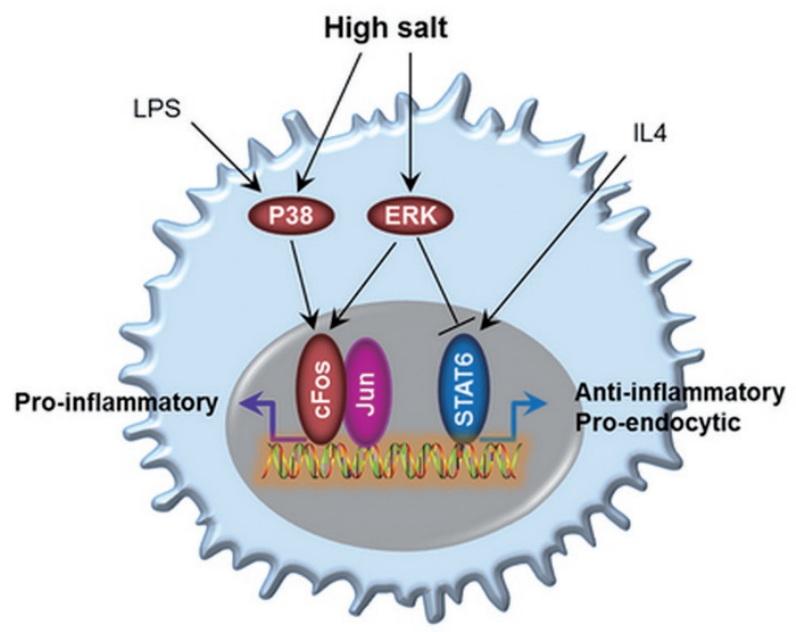

C

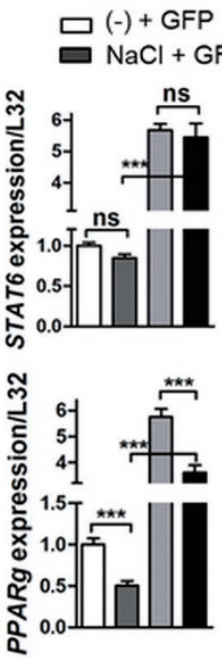

E

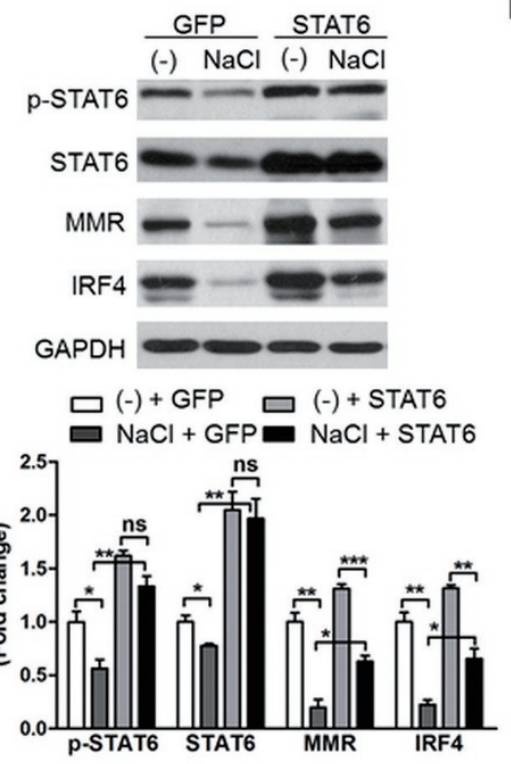

F

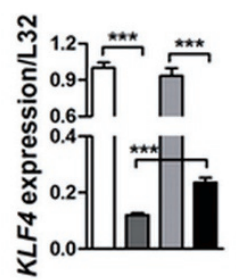

p-STATO

STAT6

ACTIN

$\square(-)+$ DMSO $\square(-)+P D$

NaCl+ DMSO $\mathrm{NaCl}+\mathrm{PD}$

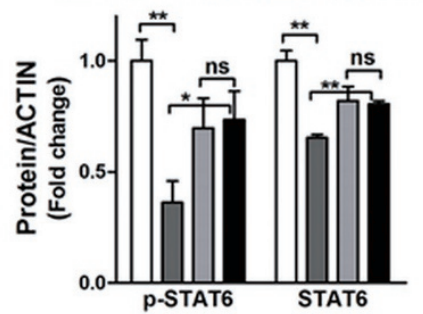

$\frac{\mathrm{DMSO}}{(-) \quad \mathrm{NaCl}} \frac{\mathrm{PD}}{(-) \quad \mathrm{NaCl}}$
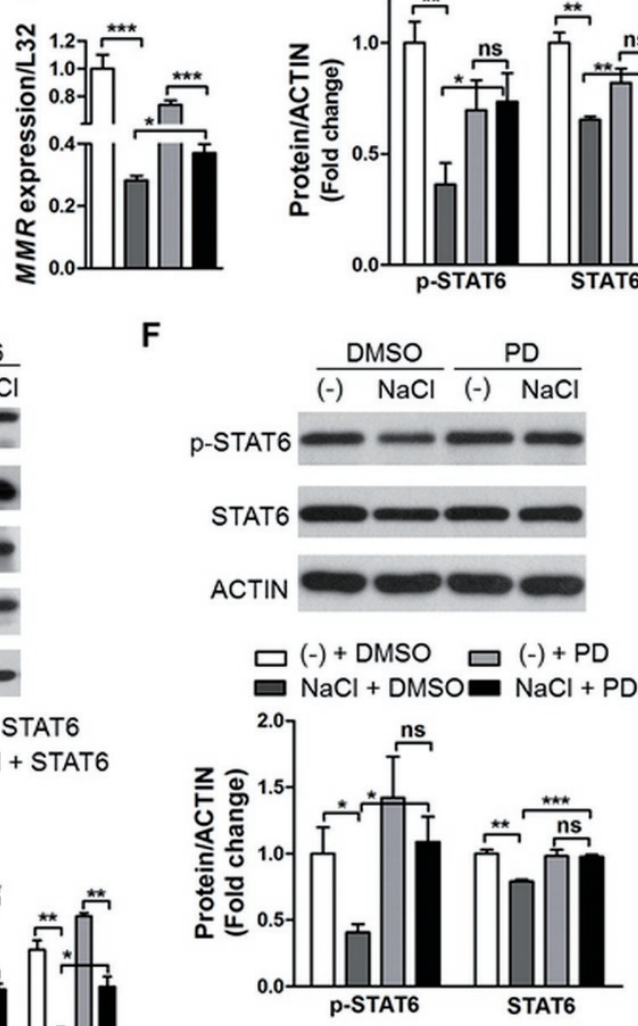

IL4 
analysis. qRT-PCR results showed that expression levels of proinflammatory genes including $C X C L 1$, chemokine (C-C motif) ligand 3 (CCL3), IL12B, CCR2, TLR4, and iNOS were significantly higher in macrophages from HSD group than those from CD group (Figure 7A). However, high salt did not affect the number of $\mathrm{CD} 11 \mathrm{~b}^{+}$cells, monocytes, or neutrophils in mouse lungs at baseline (Supplementary information, Figure S7A and S7B).

Then we explored the effects of high salt intake in LPS-challenged mice. In a mouse sepsis model induced by intraperitoneal injection of LPS, CXCL1 and CXCL2 levels were notably higher in serum from mice of the HSD group than those of the CD group (Figure 7B). Meanwhile, we measured expression of proinflammatory genes in macrophage-rich tissues including lungs, kidneys, brains, and epididymal adipose tissues. High salt significantly enhanced gene expression of CXCL1, IL6 and $i N O S$ in lungs (Figure 7C), but not in kidneys except for $i N O S$ (Supplementary information, Figure S7C), in brains (Supplementary information, Figure S7D) or epididymal adipose tissues (Supplementary information, Figure S7E). These results suggest that high salt indeed promotes LPS-induced inflammation in vivo and this is limited to particular tissues such as lungs.

To further investigate the influence of high salt on lung inflammation, a more direct acute lung injury model was established in mice inhaling aerosolized LPS. Macrophages were sorted from lungs using flow cytometry (Supplementary information, Figure S7F). As expected, pulmonary macrophages from mice in HSD group had markedly higher expression levels of proinflammatory genes (CXCL1, IL6 and CCR2) than those from $\mathrm{CD}$ group (Figure 7D). Immunofluorescence staining results showed much more $\mathrm{CD} 11 \mathrm{~b}^{+}$cells in lungs from mice in HSD group (Figure 7E and 7F), which was further verified by flow cytometry (Figure $7 \mathrm{G}$ and $7 \mathrm{H}$ ). Further analysis of subsets of $\mathrm{CD} 11 \mathrm{~b}^{+}$cells by flow cytometry showed that the number of monocytes almost doubled in lungs from mice in HSD group compared with those in $\mathrm{CD}$ group (Figure $7 \mathrm{G}$ and $7 \mathrm{H}$ ). However, the amounts of neutrophils in lungs were similar between these two groups (Figure 7G and 7H). In addition, HSD induced significant increase in numbers of leukocytes and monocytes/macrophages, but not polymorphonuclear cells (PMNs), in bronchoalveolar lavage (BAL) fluid from aerosolized LPS-treated mice (Figure 7I). Furthermore, HSD markedly increased wet weight to dry weight ratio of lungs in these LPS-challenged mice, demonstrating more severe lung edema (Figure 7J). To test whether $\mathrm{T}_{\mathrm{H}} 17$ cells were involved in this worsening of LPS-induced lung inflammation by high salt, we measured levels of IL17A in BAL fluid and serum from mice challenged with aerosolized LPS. However, high salt did not affect protein level of IL17 in BAL fluid or serum (Supplementary information, Figure S7G), implying that $\mathrm{T}_{\mathrm{H}} 17$ cells were not critical for the aggravation of lung inflammation by high salt. Taken together, high salt markedly aggravated LPS-induced lung inflammation in mice likely by increasing monocyte recruitment and macrophage-derived proinflammatory cytokines/chemokines.

\section{Discussion}

Through the current study we defined a specific macrophage activation state induced by high salt, $\mathrm{M}(\mathrm{Na})$, which is different from M(LPS) and M(IFN $\gamma)$. We identified p38/cFos/AP1, Erk1/2/cFos/AP1, and Erk1/2/STAT6 pathways as the underlying molecular mechanisms mediating the effects of high salt. High salt represented a risk factor for LPS-induced acute lung injury in mice, likely by promoting the adhesion and recruitment of monocytes in lungs and boosting proinflammatory cytokines and/or chemokines derived from monocytes and macrophages.

Figure 6 STAT6 mediates the downregulation of anti-inflammatory and proendocytic gene expression of $M(\mathrm{Na})$ as well as high-salt-induced suppression of M(IL4). (A) High salt decreases levels of p-STAT6 and total STAT6. BMDMs were treated with additional $51 \mathrm{mM} \mathrm{NaCl}$ for the indicated time periods. Levels of p-STAT6 and STAT6 were measured and actin was used as a loading control. (B) STAT6 mediates high-salt-induced reduction of anti-inflammatory and proendocytic gene expression. RAW264.7 cells with stable overexpression of STAT6 or GFP were treated with or without additional $51 \mathrm{mM} \mathrm{NaCl}$ for 6 h. (C) High-salt-induced reduction of p-STAT6 and total STAT6 depends on Erk1/2 activation. BMDMs were pretreated with $20 \mu \mathrm{M}$ PD for $1 \mathrm{~h}$ and then treated with or without additional $51 \mathrm{mM} \mathrm{NaCl}$ for $6 \mathrm{~h}$ in the presence of $20 \mu \mathrm{M}$ PD or DMSO. (D) High salt decreases levels of p-STAT6 and STAT6 in the presence of IL4. BMDMs were treated with or without additional 51 $\mathrm{mM} \mathrm{NaCl}$ in combination with $10 \mathrm{ng} / \mathrm{ml}$ IL4 for $6 \mathrm{~h}$. (E) Overexpression of STAT6 attenuates high-salt-induced suppression of M(IL4). RAW264.7 cells with stable overexpression of STAT6 or GFP were treated with or without additional $51 \mathrm{mM} \mathrm{NaCl}$ in combination with $10 \mathrm{ng} / \mathrm{ml}$ IL4 for $24 \mathrm{~h}$. (F) High-salt-induced reduction of p-STAT6 and total STAT6 depends on Erk1/2 activation in the presence of IL4. Treatment was the same as in C except that $10 \mathrm{ng} / \mathrm{ml}$ IL4 was added to all groups. (G) Proposed model of the signaling pathways that mediate the effects of high salt in macrophages. For all panels, gene expression was measured using qRT-PCR and protein expression or phosphorylation was measured using western blotting. ns, not significant; ${ }^{*} P<0.05 ;{ }^{* *} P<0.01 ;{ }^{* *} P<0.001$. 
A

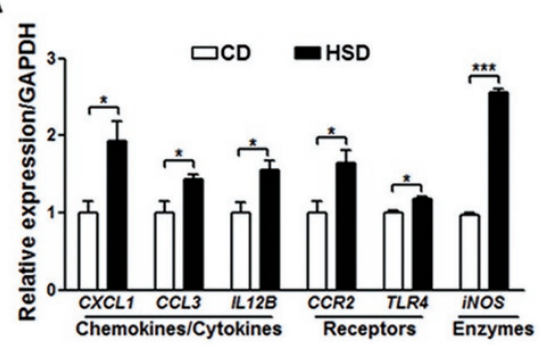

B

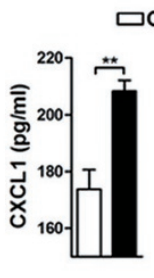

C

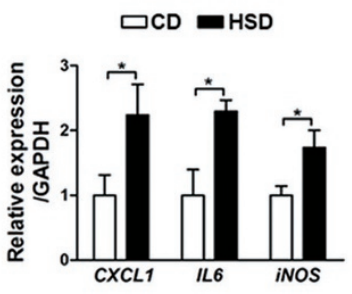

D

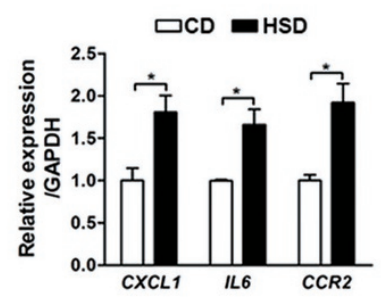

E

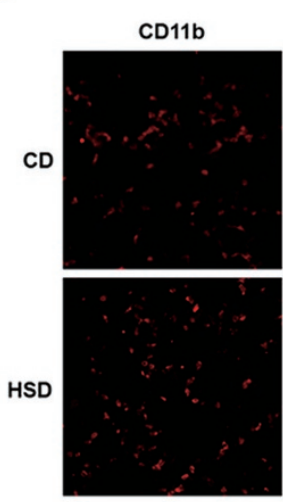

F

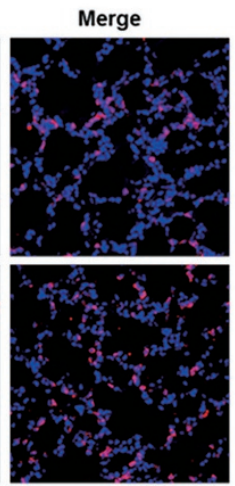

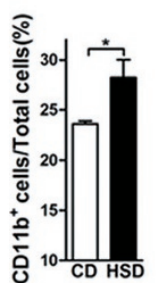

G

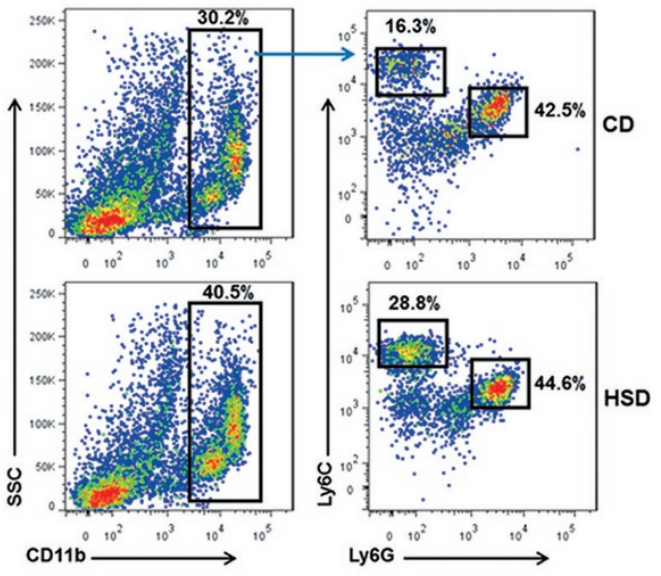

H

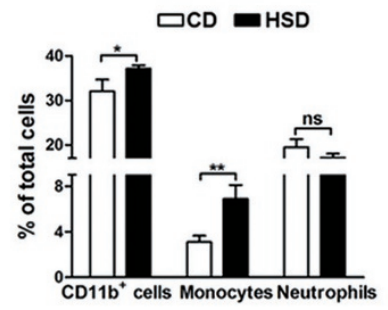

I

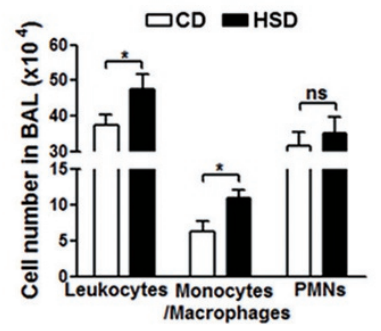

$\mathbf{J}$

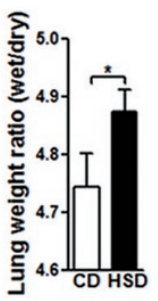

Figure 7 High salt promotes lung inflammation in mice. (A) High salt increases proinflammatory gene expression in alveolar macrophages at baseline (without LPS challenge). Alveolar macrophages were collected via BAL in mice that received chow diet and tap water (CD group) or high-salt diet (HSD) containing $4 \% \mathrm{NaCl}$ and tap water containing $1 \% \mathrm{NaCl}$ (HSD group). Expression of the indicated genes was measured using qRT-PCR $(n=4: 4)$. (B) High salt elevates serum levels of CXCL1 and CXCL2 in LPS-challenged mice. Mice were intraperitoneally injected with LPS (10 $\mu \mathrm{g}$ per $25 \mathrm{~g}$ mice) and sacrificed 1 h later. Representative results of ELISA ( $n=5: 6)$ from three independent experiments are shown. (C) High salt increases expression of proinflammatory genes in lungs from the same mice as in B. Gene expression was quantified using qRT-PCR $(n=16: 17)$. (D) High salt increases expression of proinflammatory genes in macrophages sorted from lungs after LPS challenge. Mice inhaled $1 \mathrm{mg} / \mathrm{ml}$ LPS for $35 \mathrm{~min}$ and were sacrificed $3 \mathrm{~h}$ later. F4/80 macrophages were sorted from the lungs using flow cytometry. Gene expression was quantified using qRT-PCR $(n=6: 6)$. (E, F) High salt increases CD11b ${ }^{+}$cells in mouse lungs after LPS challenge as determined by immunofluorescence staining. Mice were challenged with LPS in the same way as in $\mathbf{D}$. Representative immunofluorescence staining for CD11b (red) and images merged with DAPI (pink) are shown in $\mathbf{E}$ and quantification in $\mathbf{F}(n=6: 6)$. (G, H) High salt increases CD11 ${ }^{+}$cells and monocytes but not neutrophils in mouse lungs after LPS challenge as determined by flow cytometry. Representative flow cytometry analysis of CD11 ${ }^{+}$cells, monocytes $\left(\mathrm{CD} 11 \mathrm{~b}^{+} / \mathrm{Ly}_{6 \mathrm{G}}-/ \mathrm{Ly}_{6 \mathrm{C}^{+}}\right)$, and neutrophils $\left(\mathrm{CD} 11 \mathrm{~b}^{+} / \mathrm{Ly}_{6 \mathrm{G}} / / \mathrm{Ly}_{6 \mathrm{C}^{-}}\right)$is shown in $\mathbf{G}$ and quantification in $\mathbf{H}(n=6: 6)$. (I) High salt increases the numbers of leukocytes and monocytes/macrophages but not PMNs in BAL fluid. Mice inhaled $1 \mathrm{mg} / \mathrm{ml}$ LPS for $35 \mathrm{~min}$ and were sacrificed $6 \mathrm{~h}$ later. Leukocytes were counted using a hemocytometer. The percentages of monocytes/macrophages and PMNs were determined using Wright-Giemsa staining $(n=6: 6)$. (J) High salt aggravates lung edema in LPS-challenged mice. Mice were challenged with LPS in the same way as in I. Lung edema was indicated by wet to dry weight ratio $(n=7: 10)$. ns, not significant; ${ }^{*} P<0.05 ;{ }^{* \star} P<0.01$; ${ }^{* *} P<0.001$. 
These data indicate that high salt directly acts on macrophages to promote inflammation.

$\mathrm{M}(\mathrm{Na})$ is different from other proinflammatory macrophage activation states such as M(LPS) and M(IFN $\gamma)$. Although they have some similarities such as upregulation of proinflammatory genes and downregulation of cell cycle-related genes (data not shown) [25] as well as partially overlapped signaling pathways including activation of p38, Erk1/2, and cFos/AP1 [26], M(Na) differs from M(LPS) in several aspects. First, the proinflammatory profile of $\mathrm{M}(\mathrm{Na})$ is much more modest in comparison to that of M(LPS). This was illustrated not only by the magnitude of the gene/protein expression of proinflammatory genes but also by the range of the genes affected. For instance, high salt did not increase expression of LPS-responsive genes such as CCL4, CCL5, Cx3Cl1, ICAM1, $I R F 5, I R F 8$, and $T N F \alpha$ (data not shown). Second, $\mathrm{M}(\mathrm{Na})$ displays suppression of anti-inflammatory genes. In response to LPS, expression levels of a set of anti-inflammatory genes, including ATF3, IL10, ILIRA, SOCS1, and $I K B A$, increase as part of the mechanisms to limit prolonged inflammation [27-30]. Such increase remained $24 \mathrm{~h}$ after the initiation of LPS treatment as shown by our data or even longer as shown by others [30]. However, $\mathrm{M}(\mathrm{Na})$ showed decreased or unchanged expression of these anti-inflammatory genes. Third, the signaling pathways of $\mathrm{M}(\mathrm{Na})$ are different from those of $\mathrm{M}(\mathrm{LPS})$. There was no JNK/cJun activation in $\mathrm{M}(\mathrm{Na})$. Further, AP1 but not NFאB or IRF5 was activated in $\mathrm{M}(\mathrm{Na})$ as addressed in more details later. In addition, $\mathrm{M}(\mathrm{Na})$ has a different transcriptional profile compared with M(IFN $\gamma)$. For instance, high salt did not increase expression of IFN $\gamma$-responsive genes such as IRF5, IRF8, SOCS1 and STAT1 (data not shown).

We identified $\mathrm{p} 38 / \mathrm{cFos} / \mathrm{AP} 1$ and Erk $1 / 2 / \mathrm{cFos} / \mathrm{AP} 1$ pathways as the underlying mechanisms through which high salt induced the proinflammatory profile in macrophages. Using an inhibitor of $\mathrm{p} 38 \alpha / \mathrm{p} 38 \beta$ and myeloid-specific p38 $\alpha$-knockout mice, we demonstrated that p38 $\alpha$ was necessary for high salt to activate cFos, thus skewing macrophages toward a proinflammatory state. Given the important roles of p38 $\gamma$ and $\mathrm{p} 38 \delta$ in the inflammatory response of macrophages [31], these two isoforms may also contribute to the effects of high salt.

Previous reports have indicated that Erk1/2 may play a less essential or even contrasting role than p38 in regulating proinflammatory gene expression of LPS-stimulated macrophages $[22,32]$. We showed that Erk1/2 was similarly important in mediating the proinflammatory effects of high salt with or without LPS co-stimulation. The activation of $\mathrm{cFos}$ by high salt alone was dependent on Erk1/2 activation, which, however, was not required for the potentiation of LPS-stimulated cFos activation. Earlier studies have suggested that Erk1/2 may participate in activation of NF- $\kappa \mathrm{B}$ by promoting I $\mathrm{B}$ kinase activation and enhancing $\mathrm{I} \kappa \mathrm{B}$ degradation in LPS-stimulated macrophages [33]. Therefore, it is plausible that high salt additionally activates an Erk1/2/NF- $\mathrm{BB}$ pathway to potentiate the proinflammatory effects of LPS. It remains to be further determined whether high salt potentiates LPS-induced activation of NF- $\mathrm{KB}$ and other important proinflammatory regulators including IRF5, though we have demonstrated that these proteins and their pathways-related molecules were not affected by high salt alone in $\mathrm{M}(\mathrm{Na})$.

We demonstrated that the Erk1/2/STAT6 pathway was critical for the effects of high salt on gene and/or protein expression of both anti-inflammatory and proendocytic genes. High salt suppressed STAT6 most likely through protein degradation and inhibition of phosphorylation. High salt decreased protein level but not mRNA level of STAT6, suggesting a post-transcriptional regulatory mechanism such as protein degradation. It has been reported that STAT6 can be degraded by proteases such as calpains [34] and that activation of calpain II can be induced by $\mathrm{p}-\mathrm{Erk} 1 / 2$ [35]. Therefore, it is possible that high salt decreases total STAT6 level via promoting Erk1/2 phosphorylation and then inducing activation of calpains to degrade STAT6. It is worth mentioning that high-salt treatment resulted in more significant decrease in phosphorylation of STAT6 than total protein expression. This indicates that high salt may inhibit the phosphorylation besides causing degradation of STAT6 protein.

Our in vivo data showed that high salt aggravated lung inflammation and edema, indicating that high salt is a risk factor for acute lung injury. Monocytes are important players in sepsis-induced, high stretch mechanical ventilation-induced, and transfusion-related acute lung injuries [36-38]. Depletion of monocytes markedly attenuates the progress of these injuries [36-38]. Our results showed that high salt significantly increased monocyte recruitment in the lungs and BAL fluid of mice challenged with LPS. Such elevation of monocytes may further sensitize the lungs to the second hits such as infection and high-stretch ventilation. In addition, high salt increased the expression and secretion of proinflammatory cytokines and/or chemokines that may exacerbate lung injuries. Moreover, monocytes and macrophages are important sources of these cytokines/chemokines. The increased number of monocytes/macrophages and elevated cytokines/chemokines may ultimately contribute to the aggravation of LPS-induced alveolar-capillary barrier disruption and therefore lung edema.

High-salt-induced potentiation of monocyte recruit- 
ment may be due to the increase of their adhesion to endothelial cells in lungs. IL8 and its murine functional homologue CXCL1 markedly promote monocyte adhesion to endothelium $[39,40]$. High salt significantly elevated IL8 and/or CXCL1 in both human and mouse macrophages with or without LPS co-stimulation. In vivo, high salt potentiated gene expression and secretion of CXCL1 in mice. It is therefore conceivable that CXCL1 (and IL8) at least partially mediates the impact of high salt on the potentiation of LPS-induced accumulation of monocytes through enhancing their adhesion in lungs.

Our study added more evidence to support that macrophages are important target cells of high salt. In rats or mice fed with HSD, macrophages are necessary to maintain extracellular volume and blood pressure homeostasis through a tonicity-responsive enhancer-binding protein (also known as NFAT5)/vascular endothelial growth factor-C signaling [41, 42]. These are relatively short-term (2 weeks) effects associated with macrophage accumulation in skin and independent of inflammation. In our study, HSD treatment (7 months) sensitized mice to LPS-induced acute lung inflammation, which was demonstrated by increased monocyte accumulation in lungs and BAL fluid, elevated expression of proinflammatory genes in the whole lungs and particularly pulmonary macrophages, as well as more severe lung edema. These in vivo data and the in vitro results support direct impacts of high salt on macrophages. Sodium homeostasis is tightly regulated by the renin-angiotensin-aldosterone system [43]. To achieve sufficient sodium accumulation in vivo, we treated mice for 7 months, similar to previously reported [44].

In summary, high salt activated $\mathrm{M}(\mathrm{Na})$, potentiated M(LPS), and suppressed M(IL4). Mechanistically, p38/ cFos/AP1 and Erk1/2/cFos/AP1 pathways mediated the proinflammatory features of $\mathrm{M}(\mathrm{Na})$. p38/cFos/AP1 pathway also mediated the potentiation of M(LPS). Erk1/2/ STAT6 pathway mediated the suppression on the anti-inflammatory and proendocytic features. The in vivo data suggest that high salt is a risk factor for acute lung inflammation and edema. These results identify $\mathrm{M}(\mathrm{Na})$ as a novel macrophage activation state and suggest that macrophages may be direct target cells of high salt and may mediate the detrimental consequences of high salt consumption.

\section{Materials and Methods}

\section{Ethics statement}

All mouse experiments were carried out following the NIH Guide for the Care and Use of Laboratory Animals. All animal protocols were approved by the Institutional Review board of Institute for Nutritional Sciences, Shanghai Institutes for Biological
Sciences, Chinese Academy of Sciences (2012-AN-2).

Blood from healthy donors was obtained in accordance with protocols approved by the Institutional Review and the Ethics Board at Shanghai Ninth People's Hospital, Shanghai JiaoTong University School of Medicine (Shanghai, China). Written informed consent was obtained from each donor.

\section{Mouse strains}

Floxed p38 $\alpha$ mice [45] and floxed cFos mice [46] were crossed with LysM-Cre mice [47] to respectively generate myeloid-specific p38 $\alpha$ - and cFos-knockout mice. Erk1-knockout mice were generated as described [48]. C57BL/6 mice were purchased from Shanghai SLAC Laboratory Animal Co., Ltd (Shanghai, China). All mice were housed in specific pathogen-free (SPF) facility and fed ad libitum under the light/dark cycle of $12 \mathrm{~h}$ : $12 \mathrm{~h}$. Male C57BL/6 mice aged 5 weeks were randomized into CD group and HSD group ad libitum for 7 months, similar to that reported before [44]. Mice in CD group were fed normal diet and tap water. Mice in HSD group were fed diet containing $4 \% \mathrm{NaCl}$ and tap water containing $1 \% \mathrm{NaCl}$ as previously described $[6,7]$.

\section{Human monocyte-derived macrophages}

Peripheral blood mononuclear cells were prepared from whole blood of healthy male donors by density gradient centrifugation using Ficoll-Paque (STEMCELL technologies, 07957). Following the centrifugation, monocytes were sorted using CD14 positive selection cocktail (STEMCELL technologies, 18058) according to the manufacturer's protocol. Each $1.0 \times 10^{6}$ monocytes were cultured in a well of 12 -well plates in media containing $90 \%$ RIPA1640 (Gibco), 10\% inactivated FBS (Gibco) and $100 \mathrm{ng} / \mathrm{ml}$ human M-CSF (Peprotech, AF-300-25) without disturbance for 6 days. On the $7^{\text {th }}$ day, these monocyte-derived macrophages were treated with additional $51 \mathrm{mM} \mathrm{NaCl}$ alone or together with 100 $\mathrm{ng} / \mathrm{ml}$ LPS (Sigma-Aldrich, L4391) in the continued presence of $100 \mathrm{ng} / \mathrm{ml}$ human M-CSF.

\section{Mouse BMDMs}

L929 cells $\left(1.0 \times 10^{6}\right)$ were cultured in $55 \mathrm{ml}$ media containing 90\% RIPA1640 (Gibco) and 10\% inactivated FBS (Gibco) for 7 days. L929-conditioned media were harvested for differentiation of BMDMs. Mouse bone marrow cells were obtained from femur and tibia of 8-week male C57BL/6 mice, and then were cultured in media containing 60\% RIPA1640, 30\% L929-conditioned media, and $10 \%$ inactivated FBS in 6 -well plates at the density of $2.0 \times$ $10^{6}$ per well for 7 days. BMDMs were used for experimentation on day 8 .

\section{Establishment of RAW264.7 cells with stable overexpression} of murine STAT6

Murine STAT6 cDNA was subcloned into pHAGE-fEF1aIRES-ZsGreen plasmid (a kind gift from Professor Chenqi Xu, SIBCB, CAS). This STAT6 plasmid was then introduced into 293FT cells together with lentivirus packaging plasmids using cationic liposome (Lipofectamine 2000, Life Technologies). Media (containing lentivirus) were harvested from cells $48 \mathrm{~h}$ after transfection and filtered through $0.22-\mu \mathrm{m}$ filters. RAW264.7 cells were infected by the lentivirus and then passaged 3-4 times. ZsGreen-positive cells were sorted by flow cytometry. RAW264.7 cells with stable STAT6 overexpression were established by two rounds of GFP selection. 


\section{Alveolar macrophage isolation}

Mice were anesthetized with Avertin $(3.2 \%(\mathrm{~g} / \mathrm{ml}))$ and BAL was performed by instilling the whole lungs of each mouse with 1.3 $\mathrm{ml}$ of $1 \times$ PBS for three times. The lavage fluid was collected and combined for centrifugation to obtain alveolar macrophages.

\section{Sepsis and acute lung injury mouse models}

Mouse sepsis model was established by intraperitoneal injection of LPS (Escherichia coli 055:B5, Sigma-Aldrich, L2880) in $0.9 \%$ saline at a dose of $10 \mu \mathrm{g} / 25 \mathrm{~g}$ mouse weight. Mice were sacrificed $1 \mathrm{~h}$ later and blood, lungs, kidneys, epididymal adipose tissue, and brains were collected for analyses.

Acute lung injury was induced through inhalation of aerosolized LPS. For each independent experiment, three mice from HSD group and three mice from CD group were simultaneously exposed to $1 \mathrm{mg} / \mathrm{ml}$ aerosolized LPS for $35 \mathrm{~min}$ by placing in a custom-built chamber (Height, $15 \mathrm{~cm}$; Width, $26 \mathrm{~cm}$; Length, 26 $\mathrm{cm})$. Aerosolized LPS was delivered to the chamber through an air nebulizer. Three hours after LPS exposure, mice were anesthetized and injected with $100 \mu \mathrm{l}$ of $1 \%$ heparin to prevent blood coagulation. The mice were then systemically perfused using PBS at constant flow to wash out blood cells. Lungs were immediately taken for sorting of pulmonary macrophages, immunofluorescence staining, or flow cytometry analysis. For counting of leukocytes in BAL fluid and measurement of lung edema, mice inhaled aerosolized LPS for $35 \mathrm{~min}$ and were sacrificed $6 \mathrm{~h}$ later.

\section{Leukocyte counting}

Mice were anesthetized with Avertin $(3.2 \%(\mathrm{~g} / \mathrm{ml}))$ and BAL was performed by instilling the right lung of each mouse with 0.55 $\mathrm{ml}$ of $1 \times$ PBS for three times and the lavage fluid was collected and combined. The number of leukocytes was counted using a hemocytometer. Cytospin samples were prepared from BAL fluid using a cytospin centrifuge. Air-dried cytospin samples were used for Wright-Giemsa staining to quantify the numbers of monocytes/ macrophages and PMNs.

\section{Evaluation of lung edema}

The left lung of each mouse was collected without PBS perfusion and weighed immediately for wet weight. After 3 days of drying at $65^{\circ} \mathrm{C}$, the lung was weighed again for dry weight. Lung edema was indicated by the ratio of wet to dry weight.

\section{Immunofluorescence staining}

The post-caval lobe of each mouse was fixed in 4\% PFA for 12 $\mathrm{h}$, dehydrated using gradient glucose, and then embedded in OCT. Frozen sections of lungs $(10 \mu \mathrm{m})$ were blocked using goat serum and then stained with antibody against CD11b (ab8878, Abcam). Fluorescent signals were captured using a confocal microscope and five images were randomly chosen for quantification of $\mathrm{CD}_{11 \mathrm{~b}}{ }^{+}$cells.

\section{Flow cytometry analysis and sorting}

The left lobe, right caudal lobe, and right middle lobe of lungs from each mouse were collected together for flow cytometry analysis. The lung lobes were enzymatically digested for $30 \mathrm{~min}$ at 37 ${ }^{\circ} \mathrm{C}$ with automatic rotation to prepare single-cell suspension. The enzymatic digestion buffer comprised $2 \mathrm{mg} / \mathrm{ml}$ collagenase IV (Worthington) and $60 \mathrm{U} / \mathrm{ml}$ DNase I (Sigma-Aldrich) dissolved in HEPES buffer with $\mathrm{pH}$ 7.4. Cells were first incubated with $\mathrm{Fc}$ receptor antibodies $\left(2.4 \mathrm{G} 2, \mathrm{BD}\right.$ Biosciences) for $15 \mathrm{~min}$ at $4{ }^{\circ} \mathrm{C}$ to reduce non-specific binding, and then incubated for $40 \mathrm{~min}$ at $4{ }^{\circ} \mathrm{C}$ with antibodies including fluorescein isothiocyanate-conjugated CD11b (M1/70, eBioscience), R-phycoerythrin-conjugated (PE-conjugated) Ly6C (AL-21, BD Biosciences), allophycocyanin-conjugated Ly6G (1A8, BD Biosciences) and F4/80 (BM8, eBioscience), and PE-Cy7-conjugated CD45 (30-F11, eBioscience). After staining, cells were immediately analyzed or sorted by flow cytometry (FACSAria III, BD biosciences). Pulmonary macrophages, identified as $\mathrm{CD} 45^{+} / \mathrm{F} 4 / 80^{+}$, were sorted and then resuspended in PBS.

\section{$R N A$ sequencing}

Total RNA was extracted from human macrophages using Trizol reagent (Invitrogen) and its quality was checked using Bioanalyzer 2200 (Agilent Technologies). The RNA with RNA integrity numbers $>8.0$ was used for amplification of complementary DNA (cDNA) by Ion Total RNA-Seq Kit v2.0 (Life Technologies) according to the manufacturer's instructions. Single-end sequencing was carried out on Proton Sequencers (Life Technologies) according to Ion PI Sequencing 200 Kit v2.0 (Life Technologies). Clean reads were aligned to human genome (version: GRCH37) using the MapSplice program (v2.1.6). Original data of the sequencing were deposited into Gene expression omnibus and an accession number (GSE68482) was assigned.

\section{$q R T-P C R$}

Total RNAs from cells and tissues were extracted with TRIZOL (Invitrogen) and cDNA synthesis was accomplished using reverse transcription kits (Takara). qRT-PCR was carried out in duplicates on an ABI7900HI (Applied biosystems) using Syber Green to detect the products. Relative expression was determined via the $\mathrm{Ct}$ method and normalized to GAPDH or L32 as an internal control. Primer sequences are available upon request.

\section{Total protein extraction and western blotting analysis}

Cells were lysed with lysis buffer containing phenylmethanesulfonyl fluoride. Cell lysate was separated by SDS-PAGE and analyzed by immunoblotting. We used the following antibodies: MR (ab64693) and STAT6 (ab32520) from Abcam; COX2 (160106) from Cayman; iNOS (610328) and p-STAT6 (558241) from BD Transduction Laboratory; MGL2 (AF2835) and YM1 (BAF2446) from R\&D Systems; IRF4 (sc-6059), cFos (sc-52), and ACTIN (sc-1616) from Santa Cruz; p-p38 (4511s), p38 (9212s), p-Erk1/2 (4376s), Erk1/2 (4695), and p-cFos (5348s) from Cell Signaling Technology; GAPDH (KC-5G4) from KangChen Bio-tech; Tubulin (T6199) from Sigma-Aldrich.

\section{Measurement of cytokine levels}

Cell supernatant was collected and centrifuged to remove cell pellets. Mouse blood was allowed to clot at room temperature for $30 \mathrm{~min}$ and then centrifuged. Serum on the upper layer was collected. Cytokine levels were measured using ELISA following the manufacturer's protocols. We used the following ELISA kits: human CXCL1 (R\&D Systems, DGR00), IL8 (Genscript, L00417), CCL22 (R\&D Systems, DMD00), mouse CXCL1 (LiChen Biotech, China), CXCL2 (LiChen Bio-tech, China), and IL17 (Abcam, ab100702). 


\section{Measurement of LPS concentration}

Limulus amebocyte lysate (LAL) assay was adapted to measure LPS concentrations. Experiments were carried out in accordance with protocols from ToxinSensor Chromogenic LAL Endotoxin Assay Kit (Genscript, L00350C).

\section{TUNEL assay}

BMDMs were seeded onto culture slides and treated with additional $51 \mathrm{mM} \mathrm{NaCl}$ for $24 \mathrm{~h}$. After that, TUNEL staining was done using in Situ Cell Death Detection Kit-TMR red (Roche, 12156792910) according to the manufacturer's protocol. Fluorescent signals were captured using a confocal microscope.

\section{Statistics}

All results were presented as mean \pm SEM and analyzed using Prism software (Graph Pad). Statistical significance was determined using unpaired Student's $t$-test. A difference was considered significant if the $P$-value was $<0.05$.

\section{Acknowledgments}

This work was supported by grants from the National Basic Research Program of China (973 Program; 2012CB524900), the National Natural Science Foundation of China (91339110, 31371153, 31171133 and 31400987), the China Postdoctoral Science Foundation (2013M531225), the Postdoctoral Fellowship Program of Shanghai Institutes for Biological Sciences, Chinese Academy of Sciences (2013KIP313), and Key Laboratory of Nutrition and Metabolism, Chinese Academy of Sciences (KLNM201401). SZD was supported by the One Hundred Talents Program of the Chinese Academy of Sciences (2012OHTP06).

\section{References}

1 Pohl HR, Wheeler JS, Murray HE. Sodium and potassium in health and disease. Met Ions Life Sci 2013; 13:29-47.

2 Powles J, Fahimi S, Micha R, et al. Global, regional and national sodium intakes in 1990 and 2010: a systematic analysis of $24 \mathrm{~h}$ urinary sodium excretion and dietary surveys worldwide. BMJ Open 2013; $3: \mathrm{e} 003733$.

3 O'Donnell M, Mente A, Rangarajan S, et al. Urinary sodium and potassium excretion, mortality, and cardiovascular events. N Engl J Med 2014; 371:612-623.

4 Mozaffarian D, Fahimi S, Singh GM, et al. Global sodium consumption and death from cardiovascular causes. $N$ Engl $J$ Med 2014; 371:624-634.

5 Mente A, O'Donnell MJ, Rangarajan S, et al. Association of urinary sodium and potassium excretion with blood pressure. $N$ Engl J Med 2014; 371:601-611.

6 Kleinewietfeld M, Manzel A, Titze J, et al. Sodium chloride drives autoimmune disease by the induction of pathogenic TH17 cells. Nature 2013; 496:518-522.

7 Wu C, Yosef N, Thalhamer T, et al. Induction of pathogenic TH17 cells by inducible salt-sensing kinase SGK1. Nature 2013; 496:513-517.

8 Mattson DL. Infiltrating immune cells in the kidney in salt-sensitive hypertension and renal injury. Am J Physiol Renal Physiol 2014; 307:F499-F508.

9 Kido M, Ando K, Onozato ML, et al. Protective effect of dietary potassium against vascular injury in salt-sensitive hypertension. Hypertension 2008; 51:225-231.

10 Nagata K, Obata K, Xu J, et al. Mineralocorticoid receptor antagonism attenuates cardiac hypertrophy and failure in low-aldosterone hypertensive rats. Hypertension 2006; 47:656-664.

11 Muller S, Quast T, Schroder A, et al. Salt-dependent chemotaxis of macrophages. PLoS One 2013; 8: 73439.

12 Zhou X, Zhang L, Ji WJ, et al. Variation in dietary salt intake induces coordinated dynamics of monocyte subsets and monocyte-platelet aggregates in humans: implications in end organ inflammation. PLoS One 2013; 8:e60332.

13 Wynn TA, Chawla A, Pollard JW. Macrophage biology in development, homeostasis and disease. Nature 2013; 496:445455.

14 Davies LC, Jenkins SJ, Allen JE, Taylor PR. Tissue-resident macrophages. Nat Immunol 2013; 14:986-995.

15 Lawrence T, Natoli G. Transcriptional regulation of macrophage polarization: enabling diversity with identity. Nat Rev Immunol 2011; 11:750-761.

16 Sica A, Mantovani A. Macrophage plasticity and polarization: in vivo veritas. $J$ Clin Invest 2012; 122:787-795.

17 Xue J, Schmidt SV, Sander J, et al. Transcriptome-based network analysis reveals a spectrum model of human macrophage activation. Immunity 2014; 40:274-288.

18 Murray PJ, Allen JE, Biswas SK, et al. Macrophage activation and polarization: nomenclature and experimental guidelines. Immunity 2014; 41:14-20.

19 Martinez FO, Gordon S. The M1 and M2 paradigm of macrophage activation: time for reassessment. F1000Prime Rep 2014; 6:13.

20 Aggarwal NR, King LS, D'Alessio FR. Diverse macrophage populations mediate acute lung inflammation and resolution. Am J Physiol Lung Cell Mol Physiol 2014; 306:L709-L725.

21 Hussell T, Bell TJ. Alveolar macrophages: plasticity in a tissue-specific context. Nat Rev Immunol 2014; 14:81-93.

22 Arthur JS, Ley SC. Mitogen-activated protein kinases in innate immunity. Nat Rev Immunol 2013; 13:679-692.

23 Gilley R, March HN, Cook SJ. ERK1/2, but not ERK5, is necessary and sufficient for phosphorylation and activation of c-Fos. Cell Signal 2009; 21:969-977.

24 Tanos T, Marinissen MJ, Leskow FC, et al. Phosphorylation of c-Fos by members of the p38 MAPK family. Role in the AP-1 response to UV light. J Biol Chem 2005; 280:1884218852.

25 Ramsey SA, Klemm SL, Zak DE, et al. Uncovering a macrophage transcriptional program by integrating evidence from motif scanning and expression dynamics. PLoS Comput Biol 2008; 4:e1000021.

26 Lu YC, Yeh WC, Ohashi PS. LPS/TLR4 signal transduction pathway. Cytokine 2008; 42:145-151.

27 Ramirez-Carrozzi VR, Braas D, Bhatt DM, et al. A unifying model for the selective regulation of inducible transcription by $\mathrm{CpG}$ islands and nucleosome remodeling. Cell 2009; 138:114-128.

28 Medzhitov R, Horng T. Transcriptional control of the inflammatory response. Nat Rev Immunol 2009; 9:692-703.

29 Kinjyo I, Hanada T, Inagaki-Ohara K, et al. SOCS1/JAB is a negative regulator of LPS-induced macrophage activation. Immunity 2002; 17:583-591. 
30 Antonios JK, Yao Z, Li C, Rao AJ, Goodman SB. Macrophage polarization in response to wear particles in vitro. Cell Mol Immunol 2013; 10:471-482.

31 Risco A, del Fresno C, Mambol A, et al. p38gamma and p38delta kinases regulate the Toll-like receptor 4 (TLR4)-induced cytokine production by controlling ERK1/2 protein kinase pathway activation. Proc Natl Acad Sci USA 2012; 109:11200-11205.

32 Feng GJ, Goodridge HS, Harnett MM, et al. Extracellular signal-related kinase (ERK) and p38 mitogen-activated protein (MAP) kinases differentially regulate the lipopolysaccharide-mediated induction of inducible nitric oxide synthase and IL-12 in macrophages: Leishmania phosphoglycans subvert macrophage IL-12 production by targeting ERK MAP kinase. J Immunol 1999; 163:6403-6412.

33 Chen BC, Lin WW. PKC- and ERK-dependent activation of I kappa B kinase by lipopolysaccharide in macrophages: enhancement by $\mathrm{P} 2 \mathrm{Y}$ receptor-mediated CaMK activation. $\mathrm{Br} J$ Pharmacol 2001; 134:1055-1065.

34 Datta R, Naura AS, Zerfaoui M, et al. PARP-1 deficiency blocks IL-5 expression through calpain-dependent degradation of STAT-6 in a murine asthma model. Allergy 2011; 66:853-861.

35 Chen H, Libertini SJ, Wang Y, Kung HJ, Ghosh P, Mudryj M. ERK regulates calpain 2-induced androgen receptor proteolysis in CWR22 relapsed prostate tumor cell lines. $J$ Biol Chem 2010; 285:2368-2374.

36 Wilson MR, O'Dea KP, Zhang D, Shearman AD, van Rooijen $\mathrm{N}$, Takata M. Role of lung-marginated monocytes in an in vivo mouse model of ventilator-induced lung injury. Am J Respir Crit Care Med 2009; 179:914-922.

37 O'Dea KP, Wilson MR, Dokpesi JO, et al. Mobilization and margination of bone marrow Gr-1high monocytes during subclinical endotoxemia predisposes the lungs toward acute injury. J Immunol 2009; 182:1155-1166.

38 McKenzie CG, Kim M, Singh TK, Milev Y, Freedman J, Semple JW. Peripheral blood monocyte-derived chemokine blockade prevents murine transfusion-related acute lung injury (TRALI). Blood 2014; 123:3496-3503.

39 Gerszten RE, Garcia-Zepeda EA, Lim YC, et al. MCP-1 and IL-8 trigger firm adhesion of monocytes to vascular endothe- lium under flow conditions. Nature 1999; 398:718-723.

40 Zhou Z, Subramanian P, Sevilmis G, et al. Lipoprotein-derived lysophosphatidic acid promotes atherosclerosis by releasing CXCL1 from the endothelium. Cell Metab 2011; 13:592-600.

41 Machnik A, Neuhofer W, Jantsch J, et al. Macrophages regulate salt-dependent volume and blood pressure by a vascular endothelial growth factor-C-dependent buffering mechanism. Nat Med 2009; 15:545-552.

42 Wiig H, Schroder A, Neuhofer W, et al. Immune cells control skin lymphatic electrolyte homeostasis and blood pressure. $J$ Clin Invest 2013; 123:2803-2815.

43 Lastra G, Dhuper S, Johnson MS, Sowers JR. Salt, aldosterone, and insulin resistance: impact on the cardiovascular system. Nat Rev Cardiol 2010; 7:577-584.

44 Gao F, Liang Y, Wang X, et al. TRPV1 activation attenuates high-salt diet-induced cardiac hypertrophy and fibrosis through PPAR-delta upregulation. PPAR Res 2014; 2014:491963.

45 Engel FB, Schebesta M, Duong MT, et al. p38 MAP kinase inhibition enables proliferation of adult mammalian cardiomyocytes. Genes Dev 2005; 19:1175-1187.

46 Min L, Ji Y, Bakiri L, et al. Liver cancer initiation is controlled by AP-1 through SIRT6-dependent inhibition of survivin. Nat Cell Biol 2012; 14:1203-1211.

47 Clausen BE, Burkhardt C, Reith W, Renkawitz R, Forster I. Conditional gene targeting in macrophages and granulocytes using LysMcre mice. Transgenic Res 1999; 8:265-277.

48 Pages G, Guerin S, Grall D, et al. Defective thymocyte maturation in p44 MAP kinase (Erk-1) knockout mice. Science 1999; 286:1374-1377.

(Supplementary information is linked to the online version of the paper on the Cell Research website.)

(c) (1) () $)$ This work is licensed under the Creative Commons Attribution-NonCommercial-No Derivative Works 3.0 Unported License. To view a copy of this license, visit http:// creativecommons.org/licenses/by-nc-nd/3.0 